\title{
Protoplanetary Disk Masses from Radiative Transfer Modeling: A Case Study in Taurus
}

\author{
Nicholas P. Ballering (iD) and Josh A. Eisner (iD \\ Steward Observatory, University of Arizona, 933 North Cherry Avenue, Tucson, AZ 85721, USA; ballerin@email.arizona.edu \\ Received 2018 September 29; revised 2019 February 19; accepted 2019 February 20; published 2019 March 18
}

\begin{abstract}
Measuring the masses of protoplanetary disks is crucial for understanding their planet-forming potential. Typically, dust masses are derived from (sub-)millimeter flux density measurements plus assumptions for the opacity, temperature, and optical depth of the dust. Here we use radiative transfer models to quantify the validity of these assumptions with the aim of improving the accuracy of disk dust mass measurements. We first carry out a controlled exploration of disk parameter space. We find that the disk temperature is a strong function of disk size, while the optical depth depends on both disk size and dust mass. The millimeter-wavelength spectral index can be significantly shallower than the naive expectation due to a combination of optical depth and deviations from the Rayleigh-Jeans regime. We fit radiative transfer models to the spectral energy distributions (SEDs) of 132 disks in the Taurus-Auriga region using a Markov chain Monte Carlo approach. We used all available data to produce the most complete SEDs used in any extant modeling study. We perform the fitting twice: first with unconstrained disk sizes and again imposing the disk size-brightness relation inferred for sources in Taurus. This constraint generally forces the disks to be smaller, warmer, and more optically thick. From both sets of fits, we find disks to be $\sim 1-5$ times more massive than when derived using (sub-)millimeter measurements and common assumptions. With the uncertainties derived from our model fitting, the previously measured dust mass-stellar mass correlation is present in our study but only significant at the $2 \sigma$ level.
\end{abstract}

Key words: circumstellar matter - planetary systems

Supporting material: figure set, machine-readable tables

\section{Introduction}

Protoplanetary disks of gas and dust around young stars are the birthplaces of planets. Measuring the properties of a representative sample of these disks is necessary to interpret the diversity of observed planetary systems and constrain models of planet formation.

The masses of protoplanetary disks are arguably their most important property with regard to the number and types of planets they may form. Absolute measurements of disk masses can be compared with the masses of known exoplanets (Najita \& Kenyon 2014) or the minimum-mass solar nebula (Weidenschilling 1977; Hayashi 1981; Desch 2007) to assess whether the disks could form planets like those in our solar system. Measurements of disk masses relative to each other are also important for identifying correlations with other disk properties (e.g., Tazzari et al. 2017; Tripathi et al. 2017) and stellar properties (e.g., Andrews et al. 2013; Pascucci et al. 2016; Ansdell et al. 2017; Eisner et al. 2018).

While dust is thought to comprise only $\sim 1 \%$ of the protoplanetary disk mass (based on the dust fraction of the interstellar medium), it is the reservoir from which terrestrial planets and the cores of giant planets form. Thus, measurements of dust masses are crucial for assessing planet-forming potential. Furthermore, dust dominates the opacity of disks, meaning that observations of disks are more sensitive to the dust than to the gas component. Measurements of disk gas masses are subject to additional model-dependent complications, and disk gas-to-dust mass ratios remain uncertain (Williams \& Best 2014; Miotello et al. 2016; Bergin \& Williams 2018). In this study, we focus exclusively on measuring disk dust masses.

The dust mass of a disk is often calculated from its brightness in the (sub-)millimeter according to the analytic relation

$$
M_{\text {dust,ana }}=\frac{F_{\nu} d^{2}}{\kappa B_{\nu}\left(T_{\text {dust,ana }}\right)}
$$

(Hildebrand 1983; Beckwith et al. 1990). Here $F_{\nu}$ is the measured (sub-)millimeter flux density, $d$ is the distance to the disk from Earth, $\kappa$ is the dust opacity at the observed wavelength, and $B_{\nu}\left(T_{\text {dust,ana }}\right)$ is the Planck function at the average dust temperature. While computing dust masses with Equation (1) is common practice (e.g., Andrews \& Williams 2005; Andrews et al. 2013; Carpenter et al. 2014; Eisner et al. 2016, 2018; Pascucci et al. 2016), this method requires that specific values for the dust opacity and temperature be chosen. The relation is also predicated on the assumption that the disk is entirely optically thin to its own thermal emission at the observed wavelength. Throughout this paper, we will refer to the dust mass derived using Equation (1) as the "analytic" mass.

The dust temperature used in Equation (1) (which we will refer to as the "analytic" dust temperature) is sometimes taken to be $20 \mathrm{~K}$. Another common approach is to scale the dust temperature with the luminosity of the host star, as in the relation

$$
T_{\text {dust,ana }}=25\left(L_{\star} / L_{\odot}\right)^{1 / 4} \mathrm{~K}
$$

used by Andrews et al. (2013).

A more complete understanding of protoplanetary disks can be acquired by examining their spectral energy distributions (SEDs). The near-IR emission, or lack thereof, reveals the location of the disk's inner edge. The brightness in the mid-IR, where the disk is typically optically thick, traces the temperature of the disk surface. The shape of the SED from the mid- to far-IR indicates the vertical structure of the disk. 
The slope (spectral index) at long wavelengths can reveal the dust grain sizes, providing a more informed estimate of the dust opacity.

While first-order metrics of a disk SED-such as the spectral index between pairs of wavelengths - can serve as a basis for classification and relative comparison (e.g., Lada 1987), a model that can reproduce the entire SED is preferable for relating the SED to the underlying physical properties of the disk. The simplest commonly employed model is a "flat disk," where the dust surface density and temperature radial profiles are modeled as separate power laws. Flat-disk models, however, cannot constrain the disk's vertical structure, nor do they reflect the coupling between the disk structure and temperature. More sophisticated analytic models, such as the two-layer flared-disk model by Chiang \& Goldreich (1997), are also commonly used.

Radiative transfer modeling provides a more robust approach. In such models, photons from the central star are propagated into a specified dust distribution, defined on a cellbased grid. The temperature of the dust in each cell is computed by simulating the absorption and reemission of photons by the dust, and the simulated SED or image reflects the propagation of radiation out of the disk. This technique models the dust temperature and optical depth in a realistic manner, making it particularly useful for assessing the assumptions used in the analytic approaches. In Section 2, we employ radiative transfer models to explore the effect of various disk parameters on the observable SEDs and properties of disks that are crucial for accurately measuring their dust masses (opacity, temperature, and optical depth).

Protoplanetary disks exhibit a diversity in their mass and other properties, and insights into planet formation can be made by exploring patterns in that diversity. To do so requires analyzing a large sample of disks with a coherent modeling framework. In Section 3, we fit radiative transfer models to a large sample of disk SEDs in Taurus-Auriga. At $\sim 140 \mathrm{pc}$, Taurus is one of the nearest star-forming regions. It is frequently targeted for observation, yielding well-sampled SEDs for most of its disk-bearing members. In Section 4, we discuss the broader implications of our findings, and in Section 5, we summarize our results.

\section{Radiative Transfer Models}

\subsection{Disk Model Setup}

Our disk model is azimuthally symmetric with a radial surface density profile following

$$
\Sigma(r)=\Sigma_{0}\left(\frac{r}{r_{c}}\right)^{-\gamma} \exp \left[-\left(\frac{r}{r_{c}}\right)^{2-\gamma}\right]
$$

from an inner edge $r_{\text {in }}$ to an outer edge $r_{\text {out }}=10 r_{c}$, where $r_{c}$ is the characteristic disk size. Equation (3) is the profile predicted for a viscously accreting disk with viscosity varying as a power law with disk radius (Lynden-Bell \& Pringle 1974; Hartmann et al. 1998). We fixed the radial profile index to $\gamma=1$ for all models, as $\gamma \sim 1$ has been found by analyses of disks resolved at (sub-)millimeter wavelengths (Andrews et al. 2010; Tazzari et al. 2016). Varying $\gamma$ within reasonable bounds has a negligible effect on the SED (Woitke et al. 2016), and independently constraining $\gamma$ requires spatially resolved disk observations. The normalization, $\Sigma_{0}$, is linked to the total dust mass as

$$
\Sigma_{0}=\frac{M_{\mathrm{dust}}(\gamma-2)}{\left.2 \pi r_{c}^{2}\left[\exp \left(-\left(\frac{r}{r_{c}}\right)^{2-\gamma}\right)\right]\right|_{r=r_{\text {in }}} ^{r=r_{\text {out }}} .}
$$

When $r_{\text {in }} \ll r_{c}$ and $r_{\text {out }} \gg r_{c}, \Sigma_{0} \approx M_{\text {dust }}(2-\gamma) /\left(2 \pi r_{c}^{2}\right)$. The volume density of dust follows

$$
\rho(r, z)=\frac{\Sigma(r)}{\sqrt{2 \pi} H(r)} \exp \left[-\frac{1}{2}\left(\frac{z}{H(r)}\right)^{2}\right]
$$

with scale height

$$
H(r)=H_{100}\left(\frac{r}{100 \mathrm{au}}\right)^{\beta} .
$$

Here $H_{100}$ sets the overall vertical extent of the disk, while $\beta$, the "flaring parameter," determines how the scale height varies radially. Note that $r$ and $z$ in the preceding relations are cylindrical coordinates.

In theory, the disk vertical structure is set by hydrostatic equilibrium, so it could be determined self-consistently from the disk temperature profile computed by radiative transfer models. Indeed, some previous studies have adopted this approach (e.g., Dullemond \& Dominik 2004; Mulders \& Dominik 2012; Hendler et al. 2017). However, hydrostatic equilibrium only applies to the gas component, whereas radiative transfer calculations compute the dust temperature, so typically, the gas temperature is simply set equal to the dust temperature. Furthermore, the vertical distribution of the dust may differ from that of the gas due to, e.g., dust settling, which requires one or more additional free parameters to implement in the model. In practice, using hydrostatic equilibrium requires multiple iterations of radiative transfer calculations to find a self-consistent model, which makes the approach more computationally expensive. For these reasons, we opt to ignore the gas component and simply model the dust distribution directly.

Models of disk SEDs often use a power-law prescription for the spectral behavior of the dust opacity $\kappa(\lambda)$. While a single power law may be a good approximation of the real dust opacity at (sub-)millimeter wavelengths, it is less accurate at shorter wavelengths. Furthermore, independent parameters are often used to set the amplitude and slope of the power-law model. In reality, these parameters are correlated and determined by the more fundamental properties of the dust grains (e.g., sizes and compositions).

For our modeling, we computed the dust opacity, $\kappa(\lambda)$, with the DIANA Project Opacity Tool ${ }^{1}$ (Woitke et al. 2016). This code uses the optical constants of amorphous laboratory silicates $\left(\mathrm{Mg}_{0.7} \mathrm{Fe}_{0.3} \mathrm{SiO}_{3}\right.$; Dorschner et al. 1995) and amorphous carbon (BE sample; Zubko et al. 1996). It uses the distribution of hollow spheres method (Min et al. 2005), for which we set the "irregularity parameter" to the default value of $V_{\text {hollow }}^{\max }=0.8$. We fixed the grain composition to the default mixture of $60 \%$ silicates, $15 \%$ carbon, and $25 \%$ porosity (vacuum). The amount of carbon in protoplanetary dust is not well constrained, but based on solar system estimates, the silicate/carbon ratio is often assumed to be roughly a few (Min et al. 2011). A porosity fraction of $25 \%$ has been shown to give

\footnotetext{
1 http://dianaproject.wp.st-andrews.ac.uk/data-results-downloads/fortranpackage/
} 
good agreement with more realistic aggregate grain models (Min et al. 2016). We do not explore the effect on the disk SEDs of varying the grain composition, but this has been investigated in other studies (e.g., Miyake \& Nakagawa 1993; D'Alessio et al. 2006; Woitke et al. 2016).

The grain sizes followed a power-law distribution $n(a) \propto a^{-q}$ (where $a$ is the grain radius) from $a_{\min }$ to $a_{\max }$ with $q$ and $a_{\max }$ as free parameters and $a_{\text {min }}$ fixed to $0.05 \mu \mathrm{m}$. We computed the opacities with 100 grain size bins at 300 wavelength points. The dust opacity was assumed to be constant throughout the disk. Spatial variations in grain properties are best studied with wellresolved images of disks at multiple radio wavelengths (e.g., Pérez et al. 2015; Tazzari et al. 2016; Tripathi et al. 2018), rather than from the analysis of unresolved SEDs, as we conduct here.

We performed radiative transfer modeling with RADMC-3D (Dullemond et al. 2012) on a spherical coordinate grid. The radial grid spacing was divided into two regions in order to enhance the density of grid cells near the inner edge of the disk (as recommended by the RADMC-3D instruction manual), with the inner region from $r_{\text {in }}$ to $3 r_{\text {in }}$ and the outer region from $3 r_{\text {in }}$ to $r_{\text {out }}$. Each region had 60 radial grid steps distributed logarithmically. The grid spacing in polar angle $\theta$ was also divided into multiple regions to enhance the grid spacing near the disk midplane, with 10 grid steps from 0.1 to $1 \mathrm{rad}, 80$ grid steps from 1 to $\pi-1$, and another 10 grid steps from $\pi-1$ to $\pi-0.1$. The grid spacing was uniform in each region. Because we assumed azimuthal symmetry, we used only two cells in azimuth. The dust density in each cell was determined from Equation (5), calculated at the center of the cell.

For the stellar spectrum (the central radiation source for the radiative transfer), we used the PHOENIX "BT-settl" models (Baraffe et al. 2015) for stars $T_{\star}<7000 \mathrm{~K}$ and ATLAS9 models (Castelli \& Kurucz 2004) when $T_{\star}>7000 \mathrm{~K}$.

Each radiative transfer model was performed in two steps. In the first step, the temperature of the dust was computed in each cell. We used $10^{7}$ photons at 200 wavelengths distributed logarithmically from 0.1 to $5000 \mu \mathrm{m}$. We found that this many photons was necessary to maintain low noise in the temperature profile of the most dense disk models. We modeled the star as a spherical emitter, and we used the modified random walk algorithm. We did not include accretion heating in the disk, as this is typically only relevant in a small region of the overall disk. We also did not include heating from an external background radiation field, as previous radiative transfer studies of disks in this region have found this to have a negligible effect on the dust temperature (van der Plas et al. 2016).

In the second step, the emission from the disk was simulated, yielding the model SED. We used $10^{4}$ photons to model the SED at 200 wavelengths from 0.1 to $5000 \mu \mathrm{m}$. Scattered light from the dust in the disk was not included in the model SED, but the direct contribution of flux from the star was included.

\subsection{Exploration of Model Parameters}

To explore the effects of the model parameters, we constructed a fiducial model and varied each parameter individually from its fiducial value. The eight free parameters are summarized in Table 1. The fiducial model has $M_{\text {dust }}=10^{-4} M_{\odot}, r_{\text {in }}=0.1 \mathrm{au}$, $r_{c}=100 \mathrm{au}, H_{100}=10 \mathrm{au}, \beta=1.15, a_{\max }=10^{4} \mu \mathrm{m}, q=3.5$, and $i=40^{\circ}$. We fixed the stellar parameters to $T_{\star}=3500 \mathrm{~K}$ and $L_{\star}=0.5 L_{\odot}$.
The effect on the model SED is presented in Figure 1. Here $M_{\text {dust }}$ has little effect on the SED at short wavelengths where the disk is optically thick, but the flux density at long wavelengths scales roughly linearly with $M_{\text {dust }}$, making (sub-)millimeter observations crucial to measuring dust masses. Increasing $r_{\text {in }}$ depletes the disk of hot and warm dust near the star, thus reducing the near-IR and then mid-IR emission from the disk. An SED lacking in short-wavelength excess emission is the characteristic signature of a transition disk with a cleared inner cavity. Compared with other parameters, $r_{c}$ has a smaller influence on the overall SED, with only very small disks having a noticeable effect. Spatially resolved observations of disks are usually required to constrain the disk size. Increasing the scale height, $H_{100}$, increases the amount of the stellar radiation absorbed by the surface of the disk, increasing its temperature and thus its brightness in the infrared. A highly flared disk will intercept more flux in its outer part and less in its inner regions, making the disk brighter in the far-IR and fainter in the near-IR. A flatter (less flared) disk exhibits the opposite behavior. The disk inclination has little effect on the SED except when it is close to edge-on, in which case the central star and inner hot regions of the disk are occulted. The influence of the disk structure on the SED presented here generally agrees with the results from similar studies (e.g., Miyake \& Nakagawa 1995; D’Alessio et al. 1999; Woitke et al. 2016).

The dust grain properties $\left(a_{\max }\right.$ and $\left.q\right)$ influence the observed disk SED via the opacity spectrum, which we show in more detail in Figure 2. Smaller $a_{\max }$ values shift the entire opacity spectrum higher. They also result in a steeper slope in the (sub-)millimeter regime starting at shorter wavelengths, which translates to a steeper spectral index in the SED. Higher values of $q$ (a steeper grain size distribution and thus more small grains versus large grains) also lead to higher opacity over much of the spectrum and a steeper slope at long wavelengths. The effects of $a_{\max }$ and $q$ on the opacity spectrum agree, in general, with calculations performed using Mie theory (Miyake \& Nakagawa 1993). In the bottom panel of Figure 2, we show the effect on the opacity at $1300 \mu \mathrm{m}$ (a common wavelength at which disks are observed and their dust masses calculated) by jointly varying $a_{\max }$ and $q$. In this fairly broad range of parameter space, the opacity differed from the commonly assumed value of $2.3 \mathrm{~cm}^{2} \mathrm{~g}^{-1}$ by up to a factor of $\sim 4$.

The effect on the dust temperature of each parameter (with the other parameters fixed to their fiducial values) is shown in the top row of Figure 3. This is the mass-weighted average dust temperature,

$$
T_{\mathrm{dust}}=\frac{1}{M_{\mathrm{dust}}} \sum_{i}^{N_{\mathrm{cells}}} m_{\mathrm{dust}, i} T_{\mathrm{dust}, i}
$$

with $m_{\mathrm{dust}, i}$ the mass of dust in each cell and $T_{\mathrm{dust}, i}$ the temperature of the dust in each cell. We find that the size of the disk $\left(r_{c}\right)$ has by far the greatest effect on the disk temperature. Smaller disks can be significantly warmer than is typically assumed for $T_{\text {dust,ana }}$, in agreement with expectations and the findings of Hendler et al. (2017). Note that for this fiducial model, $T_{\text {dust, ana }} \approx 20 \mathrm{~K}$ (from Equation $(2)$ with $L_{\star}=0.5 L_{\odot}$ ).

We also tested the effect of the model parameters on the optical depth of the disk to its thermal emission. Our primary interest here was to assess the effect of optical depth on the translation from disk flux in the (sub-)millimeter to dust mass 
Table 1

Disk Model Free Parameters

\begin{tabular}{|c|c|c|c|c|c|}
\hline Parameter & Symbol & $\begin{array}{l}\text { Fiducial } \\
\text { Value }\end{array}$ & $\begin{array}{l}\text { Allowed } \\
\text { Values }\end{array}$ & $\begin{array}{l}\text { Initialized } \\
\text { Values }\end{array}$ & $\begin{array}{l}\text { Linear or } \\
\text { Logarithmic }\end{array}$ \\
\hline Dust mass & $M_{\text {dust }}$ & $10^{-4} M_{\odot}$ & $(0 \cdots \infty)$ & $\left(10^{-6} \ldots 10^{-2.5}\right) M_{\odot}$ & $\log$ \\
\hline Inner edge & $r_{\text {in }}$ & $0.1 \mathrm{au}$ & $\left(1.01 R_{\star} \cdots r_{c}\right)$ & $\left(10^{-2} \cdots 10^{2}\right) \mathrm{au}$ & $\log$ \\
\hline Characteristic size & $r_{c}$ & $100 \mathrm{au}$ & $\left(3 \cdots 10^{4}\right) \mathrm{au}$ & $\left(10^{0.8} \cdots 10^{3.5}\right) \mathrm{au}$ & $\log$ \\
\hline Scale height at $100 \mathrm{au}$ & $H_{100}$ & $10 \mathrm{au}$ & $(0 \cdots \infty)$ & $(3 \cdots 20) \mathrm{au}$ & Linear \\
\hline Flaring parameter & $\beta$ & 1.15 & $(1 \cdots \infty)$ & $(1 \cdots 1.25)$ & Linear \\
\hline Maximum grain size & $a_{\max }$ & $10^{4} \mu \mathrm{m}$ & $(0.05 \mu \mathrm{m} \cdots \infty)$ & $\left(10^{1} \cdots 10^{6}\right) \mu \mathrm{m}$ & $\log$ \\
\hline Grain size distribution index & $q$ & 3.5 & $(0 \cdots \infty)$ & $(1 \cdots 5)$ & Linear \\
\hline Inclination & $i$ & $40^{\circ}$ & $\left(0^{\circ} \ldots 90^{\circ}\right)$ & $\left(0^{\circ} \ldots 90^{\circ}\right)$ & Linear \\
\hline
\end{tabular}

(i.e., the assumption inherent in Equation (1) that the disk is optically thin). Thus, we quantify the optical depth with the metric $F_{\nu} / F_{\nu \text {,thin }}$, where $F_{\nu}$ is the flux returned by the radiative transfer model and $F_{\nu \text {,thin }}$ is the flux that would be expected if the disk were perfectly optically thin to its own thermal emission. We compute the latter as

$$
F_{\nu, \text { thin }}=\frac{\kappa}{d^{2}} \sum_{i}^{N_{\text {cells }}} m_{\text {dust }, i} B_{\nu}\left(T_{\text {dust }, i}\right) .
$$

Equation (8) is the sum of the expected flux from each cell in the model grid. The opacity and distance have been factored out of the sum because they do not vary from cell to cell. When $B_{\nu}\left(T_{\text {dust }}\right) \propto T_{\text {dust }}$ (as in the Rayleigh-Jeans regime), $F_{\nu \text {,thin }}$ is equivalent to Equation (1) (solved for $F_{\nu}$ ) using the massweighted average $T_{\text {dust }}$.

In the middle row of Figure 3, we show $F_{\nu} / F_{\nu \text {,thin }}$ at 500, 1300 , and $5000 \mu \mathrm{m}$, and we find, as expected, that the optical depth is lower at longer wavelengths. Of the eight free parameters, higher dust masses and smaller disk sizes lead to the greatest increases in optical depth (lower $F_{\nu} / F_{\nu \text {,thin }}$ ) because they result in a higher surface density. Other parameters that increase the optical depth include smaller $r_{\text {in }}$ (more mass in the dense inner region of the disk), lower $\beta$ (a more vertically compact disk), higher inclinations (more mass along the line of sight), and dust properties $\left(a_{\max }\right.$ and $\left.q\right)$ that yield a higher $\kappa$.

In the bottom row of Figure 3, we show the effect of each parameter on the ratio of $M_{\text {dust }}$ (the true mass of the dust in the model) to $M_{\text {dust,ana }}$ (the mass derived from the flux density of the model disk at $1300 \mu \mathrm{m}$ using Equations (1) and (2) and $\left.\kappa=2.3 \mathrm{~cm}^{2} \mathrm{~g}^{-1}\right)$. Dust properties yielding low values of $\kappa$ lead to higher $M_{\text {dust }} / M_{\text {dust,ana }}$. Disk properties that increase the optical depth also result in higher $M_{\text {dust }} / M_{\text {dust,ana, namely, }}$ higher $M_{\text {dust }}$, low $\beta$, and high inclinations. The disk size $\left(r_{c}\right)$ has a strong influence on both the disk temperature and optical depth, resulting in a more complicated effect on $M_{\text {dust }} / M_{\text {dust,ana }}$. Large disks are optically thin and colder than $T_{\text {dust,ana, }}$, leading to a higher $M_{\text {dust }} / M_{\text {dust,ana. }}$. Medium-sized disks have $T_{\text {dust }}$ $\approx T_{\text {dust,ana }}(20 \mathrm{~K})$, but they are slightly optically thick, and thus $M_{\text {dust }} / M_{\text {dust,ana }}$ is above unity. Small disks have hotter dust and higher optical depths-effects that act in opposing directions on $M_{\text {dust }} / M_{\text {dust,ana }}$-but the optical depth effect is stronger, so $M_{\text {dust }} / M_{\text {dust,ana }}$ increases.

\subsection{Disk Size and Dust Mass}

Since the disk size and dust mass both have significant effects on the temperature and optical depth, we next explored varying them jointly (while maintaining the other six parameters at their fiducial values). We show the results in Figure 4. The top panel again shows that the disk temperature depends primarily on disk size (although less massive disks of the same size are also slightly warmer), and that small disks are significantly warmer than assumed for $T_{\text {dust,ana. }}$ The center panel illustrates that small and/or massive disks are not entirely optically thin, even at a wavelength of $1300 \mu \mathrm{m}$, where Equation (1) is often used. The bottom panel illustrates that $M_{\text {dust }}$ can, in principle, be greater or less than $M_{\text {dust,ana, }}$ depending on the particular dust mass and disk size. The white line shows the locus where $M_{\text {dust }}=M_{\text {dust,ana. }}$ In the lower right region of the plot, this locus indicates where these masses agree for the "right" reasons-that is, the disk is truly optically thin and $T_{\text {dust,ana }}$ is an accurate measure of the average dust temperature. On the left side of the plot, however, the disks are not optically thin, so the dust masses agree only when the temperature is such that it counterbalances the optical depth effects.

In Figure 5, we show SEDs for both $F_{\nu \text {,thin }}(\lambda)$ and the full radiative transfer models for disks with a range of dust masses and disk sizes. This illustrates that the wavelength at which disks become optically thin can vary significantly, depending on these properties. For instance, a large and low-mass disk becomes optically thin in the far-IR, so Equation (1) could be applied relatively accurately to compute the disk mass from Herschel/SPIRE photometry, rather than requiring a submillimeter detection. On the other hand, a small and massive disk may not be entirely optically thin even at $\lambda \sim 5 \mathrm{~mm}$, wavelengths where the optically thin assumption is usually not questioned.

\subsection{The Spectral Index}

Also apparent from Figure 5 is that for the model disks that are not entirely optically thin in the (sub-)millimeter, the slope (spectral index) at these wavelengths is shallower than that of $F_{\nu \text {,thin }}(\lambda)$. Measuring the spectral index is important for constraining the maximum grain size to study the process of grain growth and to accurately calculate the dust mass (e.g., Beckwith \& Sargent 1991; Testi et al. 2014; Ribas et al. 2017). The spectral index can also vary with the dust composition (Pollack et al. 1994; D'Alessio et al. 2001), although we do not explore that dependence here.

In the (sub-)millimeter regime, the opacity spectrum is often approximated as a power law $\kappa(\lambda) \propto \lambda^{-\beta}$. (Note that the use of the variable $\beta$ here has no relation to the disk flaring parameter.) In the optically thin case, $F_{\nu \text {,thin }}(\lambda) \propto B_{\nu}(\lambda) \kappa(\lambda)$. Further assuming that the disk emission is in the Rayleigh-Jeans regime 

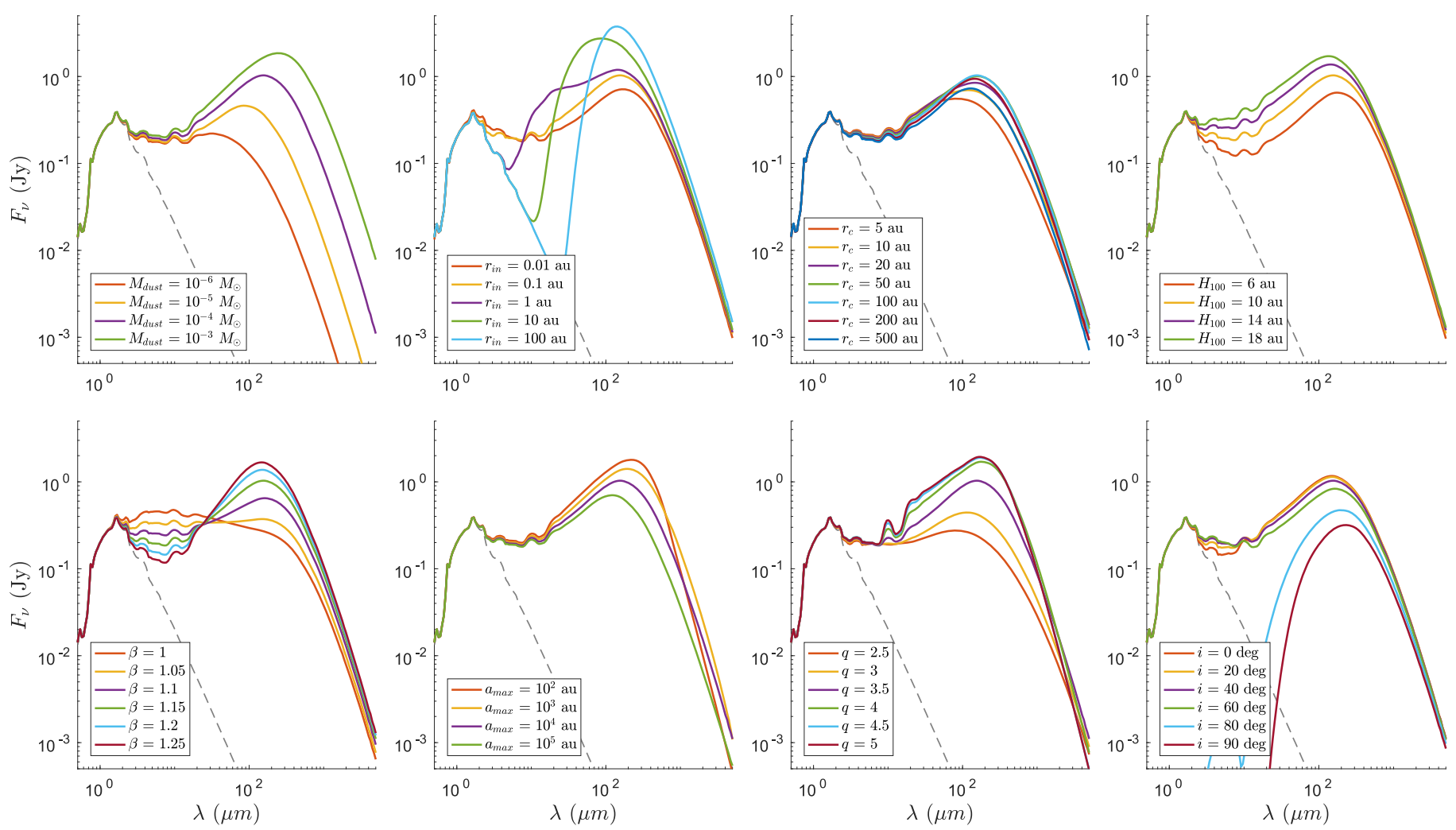

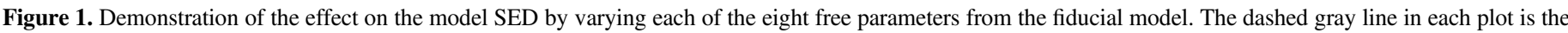
stellar photosphere.

yields $F_{\nu \text {,thin }}(\lambda) \propto \lambda^{-(\beta+2)}$. Thus, from measuring the spectral index $(\alpha)$, the slope of the opacity spectrum can be calculated as $\beta=\alpha-2$.

We used our radiative transfer models to investigate the accuracy of this method for a range of dust masses and disk sizes, and the results are shown in Figure 6. For the fiducial model dust properties, $\beta \approx 1$ (measured between wavelengths of 1 and $3 \mathrm{~mm}$ ), so if these assumptions hold, we would expect $\alpha \approx 3$. We find that both the full radiative transfer model, $F_{\nu}(\lambda)$, and the optically thin model, $F_{\nu, \text { thin }}(\lambda)$, have lower (shallower) spectral indices than this ideal expectation. For the optically thin case, the shallower spectral index is a result of the disk emission not being perfectly in the Rayleigh-Jeans regime. The discrepancy is minimized for smaller-and thus warmer-disks for which the Rayleigh-Jeans approximation is more accurate. The additional discrepancy between the optically thin spectral index and that of the full radiative transfer model is due to optical depth effects. As expected, this discrepancy is more pronounced for smaller and more massive disks. The fact that optical depth tends to decrease the spectral index can be understood by considering the limiting case of a completely optically thick disk, for which $F_{\nu, \text { thick }}(\lambda) \propto B_{\nu}(\lambda)$, and thus $\alpha \lesssim 2$. The complications that arise when interpreting the spectral index due to temperature and optical depth effects have been discussed in the literature (e.g., Beckwith et al. 1990; Testi et al. 2001; Andrews \& Williams 2005; Ricci et al. 2012). We find that radiaitve transfer models provide a valuable tool to isolate and quantify these effects.

In this section, we illustrated some of the complications involved when retrieving fundamental disk properties from observed SEDs. Fortunately, these complications can be accounted for when interpreting observations by fitting them with radiative transfer models. In Section 3, we fit models to the observed SEDs of disks in the Taurus-Auriga star-forming region.

\section{Modeling Taurus Disks}

\subsection{Target Selection and Data}

We adopted the sample of class II sources in Taurus from Andrews et al. (2013), which they argued was fairly complete. This totaled 178 systems. For the stellar properties $\left(T_{\star}, L_{\star}\right)$, we used the best-fit values from Table 4 of Andrews et al. (2013).

We discarded systems from our sample that were (1) edge-on disks or (2) disks in close binary or multiple systems. Edge-on disks obscure the star to some degree, so the inferred disk and stellar properties become correlated. In our fitting procedure, however, we keep the stellar properties fixed for a given source. Furthermore, a nonnegligible contribution to the optical and near-IR flux of edge-on systems may come from scattered light (e.g., Luhman et al. 2007), which is not included in our models. Some of the systems we discarded may actually be class I (embedded) sources, which can be mistaken for edge-on class II disks, but in either case, removal from the sample is appropriate.

We discarded close binary/multiple systems for which disk emission could not reliably be attributed to a specific star. In these cases, Andrews et al. (2013) assigned (sub-)millimeter detections to the primary components and upper limits on the flux to the secondaries. We do not adopt this procedure, as ALMA observations by Akeson \& Jensen (2014) have found that circumsecondary disks can be more massive than circumprimary disks. Only in cases where high-resolution observations (that resolve the components) show only one star 

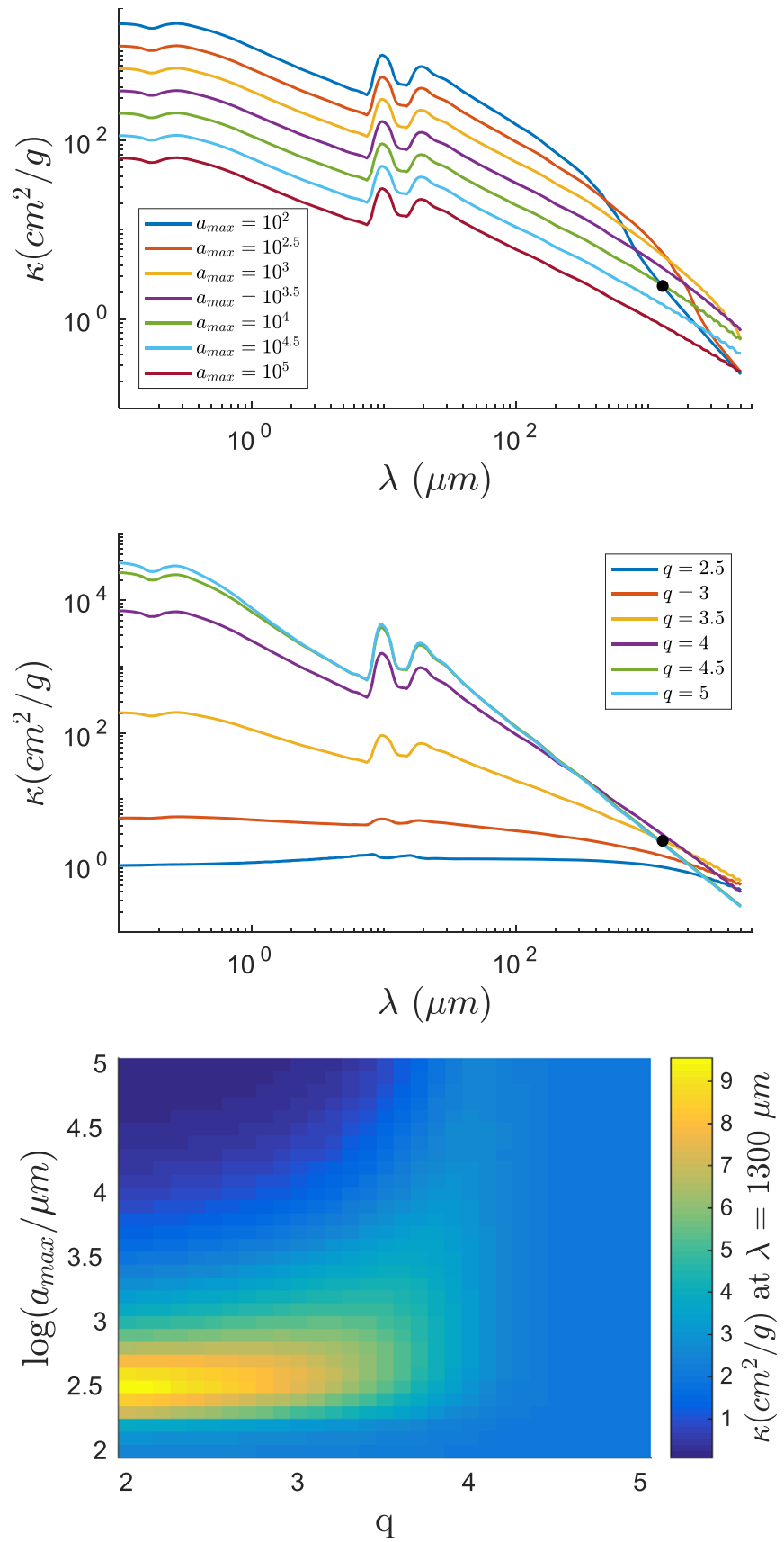

Figure 2. Top-Effect of varying the maximum grain size, $a_{\max }$, on the dust opacity spectrum with the index of the size distribution, $q$, fixed at 3.5. Middle - Same as above but varying $q$ and fixing $a_{\max }$ to $10^{4} \mu \mathrm{m}$. In both plots, $\kappa=2.3 \mathrm{~g} \mathrm{~cm}^{-2}$ at a wavelength of $1300 \mu \mathrm{m}$ is indicated with a black point. This is the value commonly assumed by previous studies. Bottom-Effect of jointly varying $a_{\max }$ and $q$ on the opacity value at $1300 \mu \mathrm{m}$.

hosting a significant disk do we assign the full measured SED to that star. In cases where multiple components host disks that are resolved at some wavelengths but not others, we simply exclude the confused data points from our fitting. We do include a few cases of known circumbinary disks, for which we modeled the central star with $L_{\star}=L_{\star, A}+L_{\star, B}$ and $T_{\star}=\left(T_{\star, A} L_{\star, A}+T_{\star, B} L_{\star, B}\right) /\left(L_{\star, A}+L_{\star, B}\right)$. We fit models to 132 disks from the original sample of 178 . Notes on specific systems - including those that we discarded from the Andrews et al. (2013) sample_-are given in the Appendix.
We used the photometry for each target provided by Andrews et al. (2013). To this, we added additional measurements from the literature, primarily at far-IR and (sub-)millimeter wavelengths. These new data are listed in Table 2. Our compiled photometry is the most complete set of SED data yet assembled for class II sources in Taurus. We dereddened the data using $A_{V}$ values for each target from Table 4 of Andrews et al. (2013) and extinction curves from McClure (2009). Total uncertainties were computed from the combination in quadrature of the statistical and calibration uncertainties. To ensure that no data point was weighted too highly in our fitting, any point with a final uncertainty of $<5 \%$ was scaled up to $5 \%$. We excluded some data points from the fitting, typically those at UV-to-visible wavelengths that showed an excess above the photosphere model. This excess luminosity is likely due to accretion, which is not accounted for in our models. The reasons for excluding other measurements are described for individual targets in the Appendix.

\subsection{SED Fitting Procedure}

We fit models to the SED of each target using the Markov chain Monte Carlo (MCMC) software package emcee (Foreman-Mackey et al. 2013). We ran 300 walkers in parallel for each target. The model generation and fitting was run on the University of Arizona High Performance Computing system.

We varied the eight free parameters introduced in Section 2. For parameters that were likely to vary by more than an order of magnitude, we used the base-10 log of the parameter in the fitting. The starting parameters for each walker were selected randomly from a uniform distribution, as noted in Table 1.

The natural log of the likelihood (the goodness-of-fit metric used by emcee) was computed as

$$
\begin{aligned}
\ln P= & -\frac{1}{2} \sum_{i}^{N_{\mathrm{det}}}\left(\frac{\log F_{\nu, \text { model }, i}-\log F_{\nu, \mathrm{det}, i}}{\sigma_{\mathrm{det}, i} /\left(F_{\nu, \mathrm{det}, i} \ln 10\right)}\right)^{2} \\
& +\sum_{j}^{N_{\mathrm{ul}}} \ln \left[\frac{1}{2} \operatorname{erfc}\left(\frac{\log F_{\nu, \text { model }, j}-\log F_{\nu, \mathrm{ul}, j}}{\sqrt{2} \log F_{\nu, \mathrm{ul}, j}}\right)\right],
\end{aligned}
$$

where $F_{\nu \text {,model }}$ are the model flux density values interpolated onto the observed wavelengths, and erfc is the complementary error function. The first sum in Equation (9) is over the detected flux density measurements and equivalent to the usual $\chi^{2}$ metric. The second sum is over the nondetection measurements ( $F_{\nu, \mathrm{ul}, j}$ is the $1 \sigma$ upper limit) and based on the formalism by Sawicki (2012). The fit, as indicated in Equation (9), was performed on the log of the flux densities. We found that, in practice, Equation (9) is weighted toward fitting the detections, with a comparatively small penalty induced when models violated upper-limit measurements. For some systems, this turned out to be beneficial, as there were measured detections at higher flux densities than upper limits reported at the same wavelengths, and it was our preference that the fits should prioritize the detections.

Parameters were bounded to the ranges given in Table 1. When emcee selected a model with one or more parameters outside of these ranges, the radiative transfer was not performed, and $\ln P$ was set to $-\infty$. We chose these bounds to be as nonrestrictive as possible, i.e., not influenced by prior modeling of disks or theoretical predictions. We examined the distributions of the model parameters versus the steps of the 

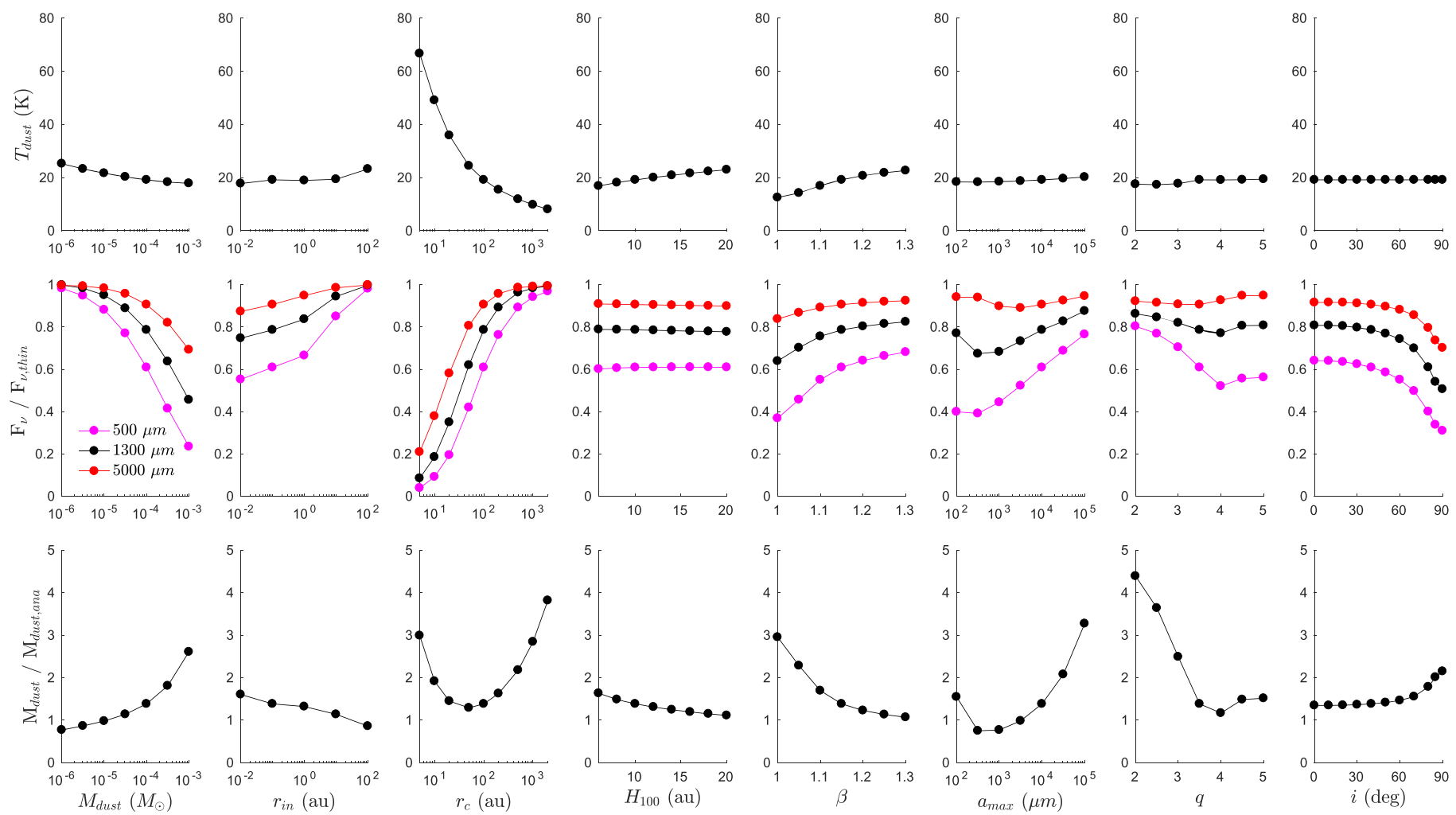

Figure 3. Effect of each of our eight disk free parameters (varied individually from the fiducial model) on the average dust temperature ( $T_{\text {dust }}$; top row), $F_{\nu} / F_{\nu, t h i n}$ at three different wavelengths (middle row), and $M_{\text {dust }} / M_{\text {dust,ana }}$ (bottom row). Here $T_{\text {dust }}$ is computed from Equation (7), $F_{\nu, \text { thin }}$ is computed from Equation (8), and $M_{\text {dust,ana }}$ is computed from Equation (1).

MCMC to identify where the results converged, and we discarded all previous steps in the chains. The remaining steps defined the posterior set of models used for our analysis.

\subsection{Disk Size Constraint}

In Section 2, we showed that the disk size has a large influence on the dust temperature and optical depth and thus on the derived dust mass. However, we also showed in Figure 1 that the disk size has only a minor effect on the observed SED and thus is difficult to constrain from fits to SEDs alone.

With this in mind, we performed our fitting of each target again, the second time with an external constraint on the disk size imposed. This constraint was the relation between disk size and submillimeter brightness established by Tripathi et al. (2017) from spatially resolved observations of disks at $880 \mu \mathrm{m}$, specifically,

$$
\log R_{\text {eff }}=(2.12 \pm 0.05)+(0.5 \pm 0.07) \log F_{\nu} .
$$

Here $R_{\text {eff }}$ is the radius of the (inclination-deprojected) disk image that encompasses $68 \%$ of the total flux density at $880 \mu \mathrm{m}$. The relation was derived using disks from Taurus and Ophiuchus, but many of our targets were not included, so we could not adopt individual disk sizes directly.

For each model SED generated during our fitting process, we also generated a model disk image ${ }^{2}$ at $880 \mu \mathrm{m}$ from which we computed $R_{\text {eff }}$. We compared this with the $R_{\text {eff }}$ expected from Equation (10) based on the model total flux density at $880 \mu \mathrm{m}$, and we computed the $\chi^{2}$ metric with the uncertainty factor

$\overline{2}$ The model image was made with RADMC-3D on a $512 \times 512$ pixel grid using $10^{5}$ photons. calculated from combining in quadrature the uncertainties on the slope and intercept of Equation (10) with 0.2 dex of scatter (Tripathi et al. 2017). We then added this $\chi^{2}$ as an extra term to the fit metric given by Equation (9).

However, this alone would have resulted in the disk size constraint influencing $\ln P$ with approximately the same weight as a single photometric data point in the SED fit, so we opted to increase its weight to be of equal order to the entire SED fit. We examined the magnitudes of the size constraint $\chi^{2}$ terms compared with the SED fit $\ln P$ terms in the posterior set of models from the initial fitting (without the size constraint included in the fitting). The factor by which to increase the weighting was the ratio of the median of the latter to the median of the former. This extra weighting factor was determined for each target individually and then applied when running the second fit (with the size constraint included).

In Figure 7, we illustrate the effect of including the size constraint in the fitting. Both panels plot $R_{\text {eff }}$ versus $F_{\nu}$ at $880 \mu \mathrm{m}$, and the red line shows the size constraint itself (Equation (10)). The top panel shows the unconstrained results for reference. The majority of disks tend to be larger than the external constraint would predict, and the range of disk sizes in the posterior set of models for each target is large. The bottom panel shows the results with the size constraint implemented. The disks are overall smaller than without the size constraint, and they have smaller ranges of sizes. Most disks fall onto the constraint, although the faintest disks exhibit a worse agreement with the trend. Tripathi et al. (2017) measured the relation with disks as faint as $\log \left(F_{\nu} / \mathrm{Jy}\right) \approx-1.5$, and Andrews et al. (2018) recently confirmed that it holds to disks that are an order of magnitude fainter. Nevertheless, our application of the size constraint to the faintest disks in our sample still represents 

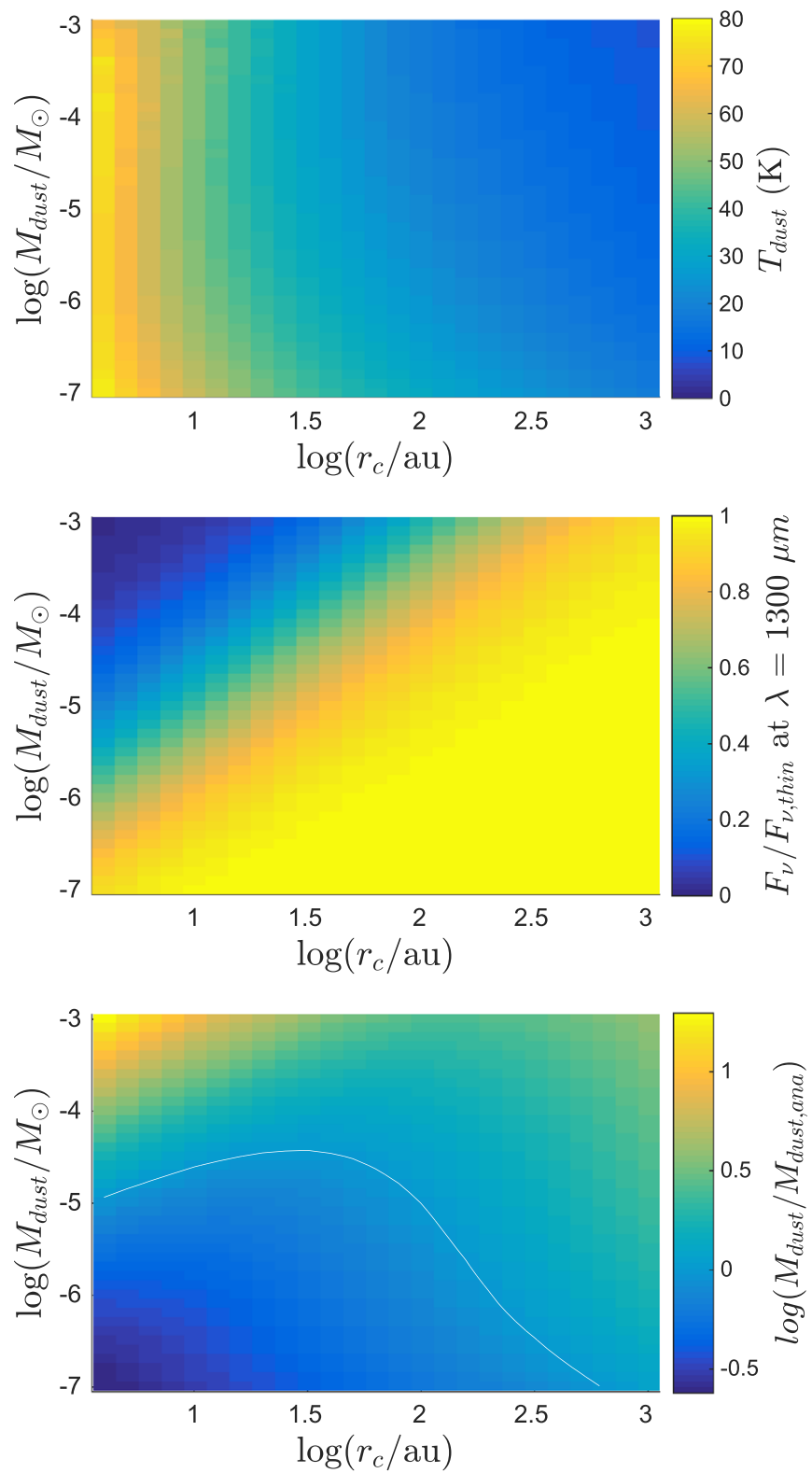

Figure 4. Effect of jointly varying the dust mass and disk size while holding other disk parameters fixed to the fiducial values on the average dust temperature ( $T_{\text {dust }}$; top), the optical depth (as described by $F_{\nu} / F_{\nu \text {,thin }}$ at $1300 \mu \mathrm{m} ;$ middle), and $M_{\text {dust }} / M_{\text {dust,ana }}$ (bottom). The white line in the bottom panel marks where the ratio is unity.

an extrapolation of the trend. The most significant outliers above the trend include V410 X-ray 7 AB, J04334171 +1750402, J04403979+2519061 AB, V819 Tau, and JH 56. The SEDs of these sources suggest that they are transition disks (or, in the case of JH 56, perhaps a cold debris disk). The need for a deficit of warm dust does not allow their SEDs to be fit with disks as small as the external constraint would require.

\subsection{Results}

The results of the MCMC fits are given in Tables 3 (without the size constraint) and 4 (with the size constraint). Each quoted value is the median of the posterior sample of models, with uncertainties spanning the 5.9-84.1 percentiles of the sample. We show the fits to the SEDs in Figure 8. Models shown in blue were fit without the size constraint, while those shown in orange were fit with the size constraint. We were not able to achieve adequate fits to five of our targets (DH Tau A, $\mathrm{J} 04213459+2701388$, J04330945+2246487, UX Tau A, and V892 Tau AB). We exclude these targets from our demographic analysis, note them with an asterisk in Figure 8 and Tables 3 and 4, and discuss them further in the Appendix.

Figure 9 shows the distributions of the median values of the eight free parameters plus $\kappa, T_{\text {dust }}, F_{\nu} / F_{\nu \text {,thin }}$, and $\log \left(M_{\text {dust }} / M_{\text {dust,ana }}\right)$ from the fits both with and without the size constraint. With the size constraint, the disks are systematically smaller and warmer, and few are entirely optically thin. Without the size constraint, the $F_{\nu} / F_{\nu \text {,thin }}$ distribution peaks around 1 with a tail toward more optically thick disks. The other parameters show no significant difference when fitting with or without the size constraint. The dust opacities show a range of values, but the distribution is strongly peaked near the oftenassumed value of $2.3 \mathrm{~cm}^{2} \mathrm{~g}^{-1}$. The dust masses of most disks found from radiative transfer modeling are systematically higher than $M_{\text {dust,ana }}$ by a factor of $\sim 1-5$. We note that other detailed SED modeling studies have also found dust masses larger than predicted by $M_{\text {dustana; }}$ e.g., Maucó et al. (2018) found $M_{\text {dust }} / M_{\text {dust,ana }}$ of $\sim 3$ and $\sim 6$ for two disks they modeled.

\section{Discussion}

\subsection{Fidelity of Previous Assumptions}

Our modeling allows us to evaluate the assumptions that are typically employed when computing disk dust masses using Equation (1). The median values of the dust opacities (at $1300 \mu \mathrm{m}$ ) peak near the assumed value of $2.3 \mathrm{~cm}^{2} \mathrm{~g}^{-1}$ (Figure 9), so this assumption is warranted. However, we find from our fitting that the uncertainty on $\kappa$ remains a significant source of uncertainty on the dust masses. Measurements at additional (sub-) millimeter wavelengths can reduce this uncertainty, and imposing independent constraints on the dust properties (e.g., from theoretical expectations) would help as well.

Radiative transfer models calculate the temperature of a disk in a realistic manner given the dust density distribution and the luminosity and spectrum of the central star, so they are well suited to test the common prescriptions for assigning a dust temperature. In Figure 10, we plot $T_{\text {dust }}$ versus $L_{\star}$ compared with the relation for $T_{\text {dust,ana }}$ given in Equation (2) and used by Andrews et al. (2013). For our fits without the size constraint, the dust temperatures tend to increase with $L_{\star}$ and are generally consistent with the $T_{\text {dust,ana }}$ relation, although the uncertainties on the dust temperatures are quite large owing to the large range of disk sizes. For the fits with the size constraint, $T_{\text {dust }}$ also increases with $L_{\star}$, but the absolute temperatures overall are higher than $T_{\text {dust,ana }}$ because, as we showed in Figures 7 and 9, the size constraint forces disks to be smaller.

Radiative transfer models are also well suited to test the assumption inherent in Equation (1) that disk (sub-)millimeter emission is entirely optically thin. We find that when disk sizes are independently constrained, very few of them remain optically thin. Even the model fits where the disk sizes are not constrained (and the disks tend to be larger), the disks are not all optically thin. Thus, the optically thin assumption has the effect of systematically underestimating dust masses for a sample of disks.

\subsection{Planet-forming Potential}

The mass of dust in protoplanetary disks is commonly used as an indicator of their planet-forming potential. However, 

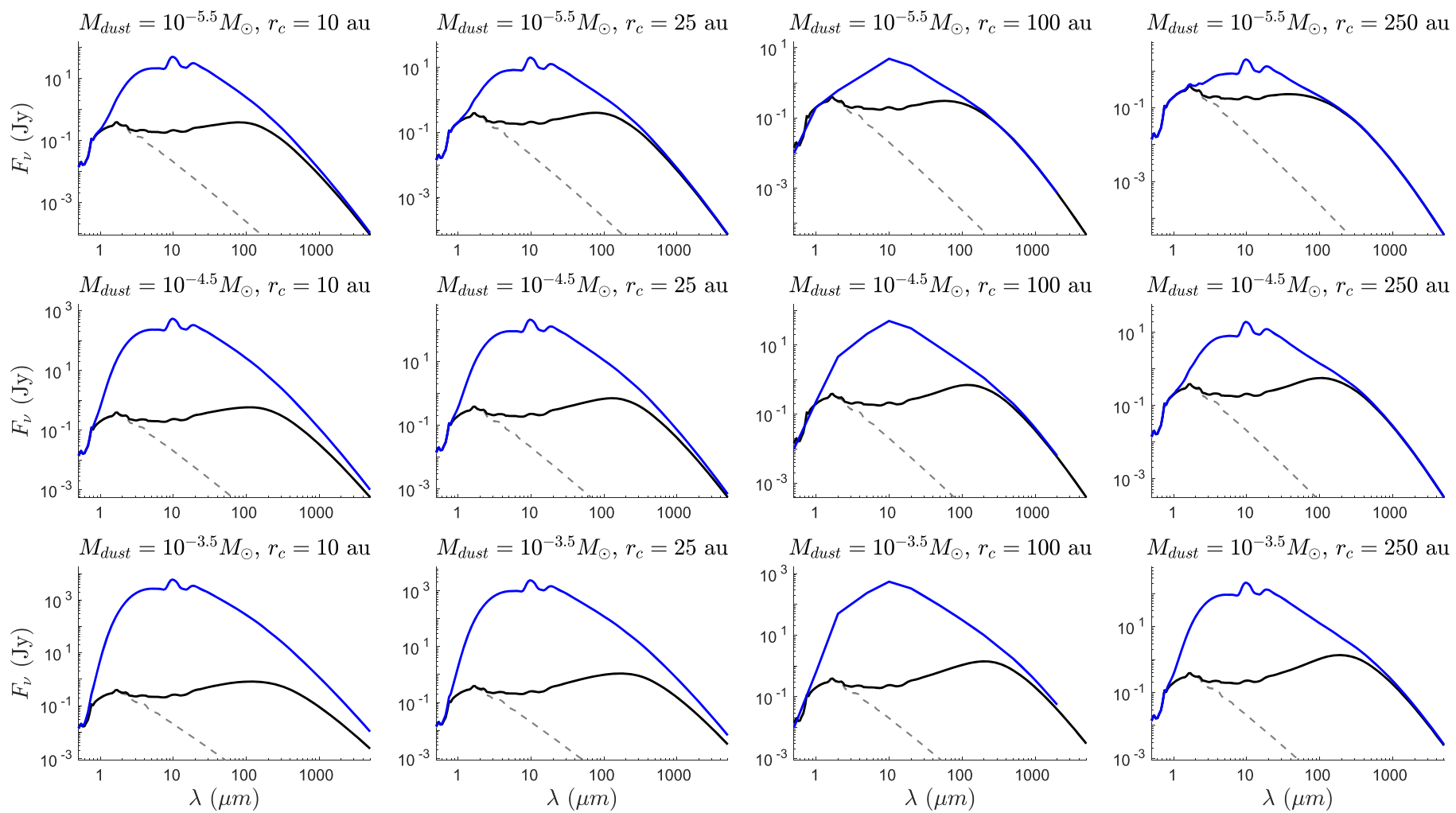

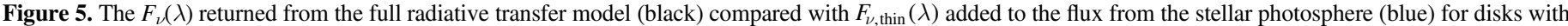
a variety of dust masses (rows) and disk sizes (columns). The gray dashed line in each plot is the stellar photosphere.
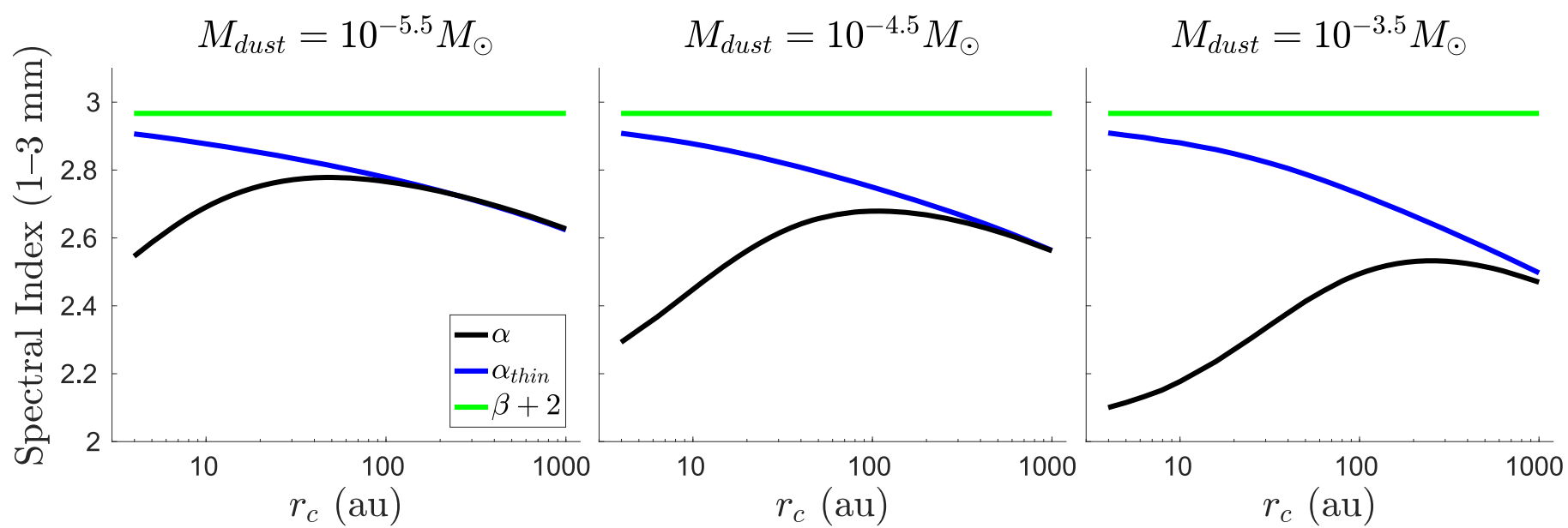

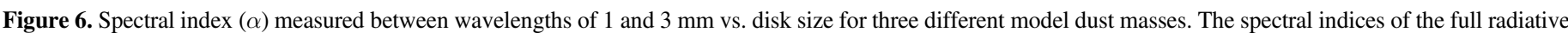

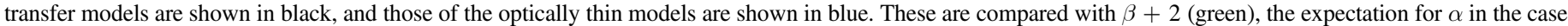

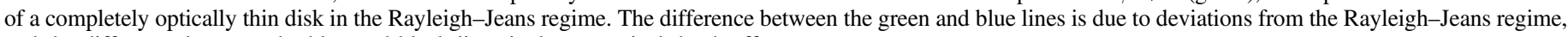
and the difference between the blue and black lines is due to optical depth effects.

ensembles of disk dust masses measured using Equation (1) are in tension with the masses of planets seen in mature planetary systems (Najita \& Kenyon 2014). Too few disks have enough dust mass to form the observed planets. A plausible solution is that the formation of planetesimals (and possibly planets) proceeds rapidly, such that a significant amount of solid mass has already been sequestered in larger bodies by the time disks reach the class II stage. The detection of annular gaps (thought to be opened by planets) in the disks of the young HL Tau system (ALMA Partnership et al. 2015) and at least one embedded class I system (Sheehan \& Eisner 2018) support this explanation. Our results offer another potential solution to this tension: that Equation (1) systematically underestimates dust masses.

To quantify the planet-forming potential of our derived dust masses, we plot their cumulative distribution in Figure 11. We compare the dust masses to a benchmark value of $10^{-4} M_{\odot}$, a common estimate for the minimum-mass solar nebula. This value tacitly assumes a gas-to-dust ratio of 100 . We find that $18 \%$ of the systems in our Taurus sample have $M_{\text {dust, ana }}>10^{-4} M_{\odot}$, whereas $28 \%$ and $31 \%$ have $M_{\text {dust }}>10^{-4} M_{\odot}$ from the radiative transfer fits with and without the size constraint, respectively. The fraction of disks with dust masses of at least the minimum-mass solar 
Table 2

Additional SED Data

\begin{tabular}{lrccccc}
\hline \hline Target & $\lambda$ & $\begin{array}{c}F_{\nu} \\
(\mathrm{mJy})\end{array}$ & $\begin{array}{c}\text { Stat } \sigma \\
(\mathrm{mJy})\end{array}$ & $\begin{array}{c}\text { Cal } \sigma \\
(\%)\end{array}$ & Instrument & References \\
\hline AA Tau & 70 & 1172.60 & 37.00 & 5 & Herschel/PACS & PACS Point Source Catalog \\
AA Tau & 100 & 1031.00 & 33.60 & 5 & Herschel/PACS & PACS Point Source Catalog \\
AA Tau & 160 & 1213.40 & 76.10 & 5 & Herschel/PACS & PACS Point Source Catalog \\
AA Tau & 250 & 1103.50 & 38.00 & 4 & Herschel/SPIRE & SPIRE Point Source Catalog \\
AA Tau & 350 & 917.90 & 44.10 & 4 & Herschel/SPIRE & SPIRE Point Source Catalog \\
AA Tau & 500 & 624.10 & 38.10 & 4 & ALMA & SPIRE Point Source Catalog \\
AA Tau & 1060 & 106.10 & 0.54 & 20 & ALMA & Loomis et al. (2017) \\
AA Tau & 1150 & 86.60 & 0.53 & 20 & SMA & Williams \& Best (2014) \\
AA Tau & 1300 & 54.80 & 0.70 & 20 & & \\
\hline
\end{tabular}

Note. See Marton et al. (2017) for details regarding the PACS Point Source Catalog and Schulz et al. (2017) for details regarding the SPIRE Point Source Catalog. (This table is available in its entirety in machine-readable form.)
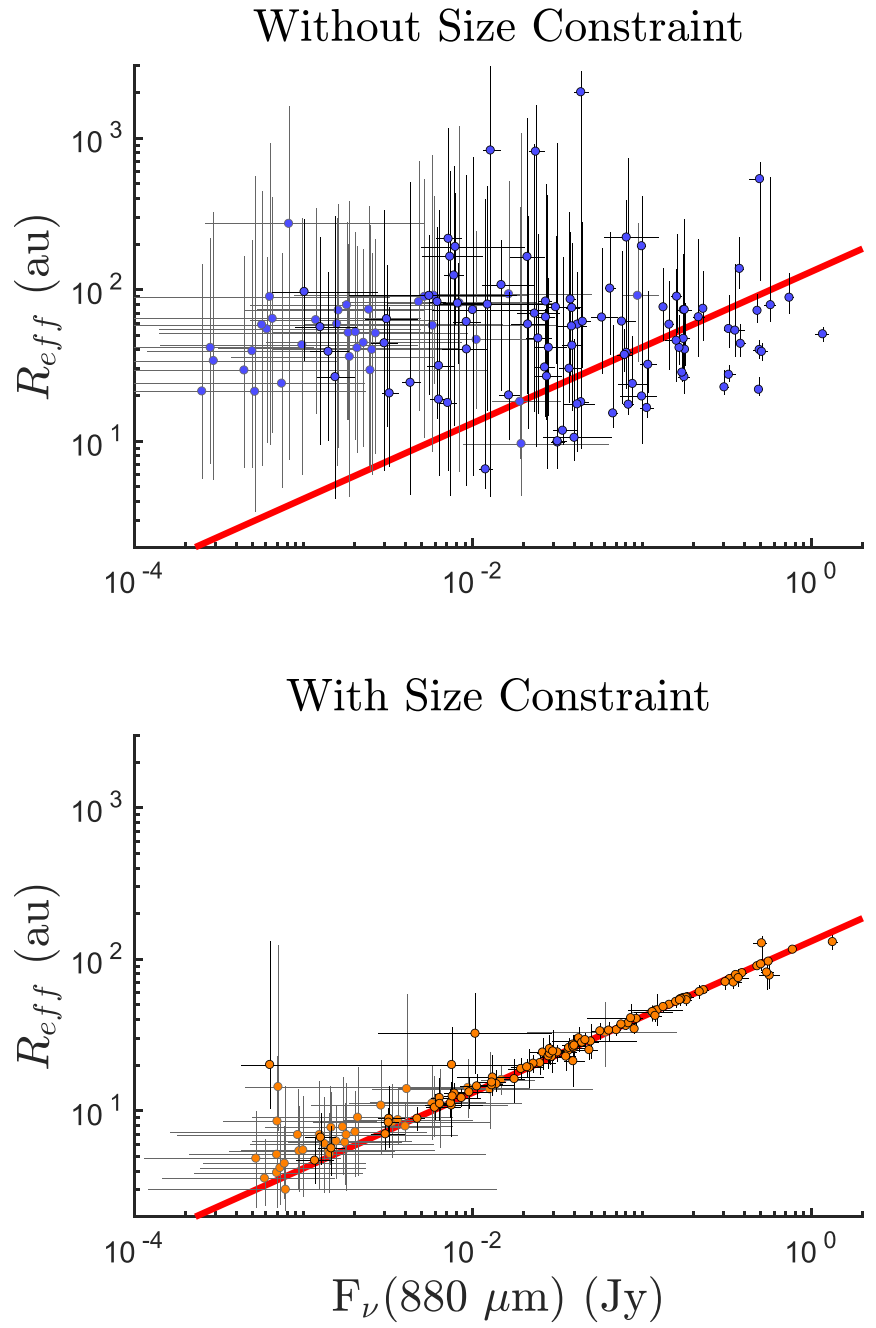

Figure 7. Effect of implementing the external size constraint in the fitting. Both panels show $R_{\text {eff }}$ vs. $F_{\nu}$ at $880 \mu \mathrm{m}$, with the red line depicting the constraint (Equation (10)). The top panel shows the results of the fitting without the constraint included, and bottom shows those with the constraint. The points outlined in black and with black error bars are systems with detections at $\lambda>500 \mu \mathrm{m}$, while those in gray have no measurements or only upper limits at $\lambda>500 \mu \mathrm{m}$.

nebula derived from our radiative transfer modeling are in agreement with the inferred occurrence rate for giant planets around FGK stars of $\sim 25 \%$ (Clanton \& Gaudi 2014).

\subsection{Dust Mass versus Stellar Mass}

Trends have been observed between the disk submillimeter flux density and stellar mass and between the disk dust mass and stellar mass for disks in Taurus (Andrews et al. 2013; WardDuong et al. 2018) and other regions (e.g., Pascucci et al. 2016). Here we look for these same trends in the results of our radiative transfer model fits to Taurus disk SEDs. As was done in the previous works, we used power-law fits to search for the correlations, i.e., $\log \left(F_{\nu} / \mathrm{Jy}\right)=A+B \log \left(M_{\star} / M_{\odot}\right)$ and $\log \left(M_{\text {dust }} / M_{\odot}\right)=A+B \log \left(M_{\star} / M_{\odot}\right)$. The disk flux was taken at a wavelength of $1300 \mu \mathrm{m}$. We looked for these correlations only using the model fits with the size constraint implemented.

Since we have additional information provided by our MCMC fitting routines, we are able to improve upon previous work in a couple of important ways. First, we did not use upper limits on the disk flux density or dust mass. This is because, even for targets without submillimeter detections, our models yielded dust masses and flux densities based on fits to the SED data that were available. Second, previous studies treated the uncertainties in their quantities as Gaussian errors and used a fitting algorithm appropriate for that assumption. We opted for a Monte Carlo approach to sample from the posterior set of models resulting from the MCMC fit to each target. We performed 10,000 samples. For each sample, we drew one value of our parameter of interest $\left(F_{\nu}\right.$ or $\left.M_{\text {dust }}\right)$ and one value of $M_{\star}$ for each target. We used the $M_{\star}$ values from Table 3 of Andrews et al. (2013), which gives three different values for each target, derived using three different stellar evolution models. We performed the fit three times, once for each of these $M_{\star}$ determinations, as was done by Andrews et al. (2013). We sampled the $M_{\star}$ values by assuming that the confidence intervals described an asymmetric Gaussian distribution (the given confidence intervals were often not symmetric). We did this by first taking a 50\% chance of the value being above or below the central value, then determining the magnitude of the deviation from that central value by sampling from a Gaussian with a standard deviation being the confidence interval from the appropriate side of the distribution.

With sample values selected for each target, the best-fit values of $B$ and $A$ were computed for the power-law fit as

$$
B=\frac{\overline{\log \left(M_{\star}\right) \cdot \log \left(M_{\text {dust }}\right)}-\overline{\log \left(M_{\star}\right)} \cdot \overline{\log \left(M_{\text {dust }}\right)}}{\overline{\log \left(M_{\star}\right)^{2}}-\overline{\log \left(M_{\star}\right)^{2}}}
$$


Table 3

Fit Results (without Size Constraint)

\begin{tabular}{|c|c|c|c|c|c|c|c|c|c|c|c|c|}
\hline Target & $\log \left(M_{\text {dust }} / M_{\odot}\right)$ & $\log \left(r_{\text {in }} / \mathrm{au}\right)$ & $\log \left(r_{c} / \mathrm{au}\right)$ & $H_{100}(\mathrm{au})$ & $\beta$ & $\log \left(a_{\max } / \mu \mathrm{m}\right)$ & $q$ & $i$ (deg) & $\kappa\left(\mathrm{cm}^{2} \mathrm{~g}^{-1}\right)$ & $T_{\text {dust }}(\mathrm{K})$ & $F_{\nu} / F_{\nu, \text { thin }}$ & $M_{\text {dust }} / M_{\text {dust,ana }}$ \\
\hline AA Tau & $-4_{-0.129}^{+0.16}$ & $-0.957_{-0.17}^{+0.1}$ & $1.74_{-0.206}^{+0.461}$ & $10.3_{-1.05}^{+1.95}$ & $1.12_{-0.0157}^{+0.0184}$ & $3.26_{-0.369}^{+0.365}$ & $3.27_{-0.395}^{+0.259}$ & $29.9_{-8.21}^{+8.86}$ & $4.11_{-1.07}^{+1.43}$ & $24.9_{-8.17}^{+6.26}$ & $0.553_{-0.144}^{+0.224}$ & $1.02_{-0.25}^{+0.447}$ \\
\hline $\mathrm{AB}$ Aur & $-3.84_{-0.177}^{+0.0525}$ & $-0.617_{-0.0489}^{+0.0456}$ & $2.9_{-0.565}^{+0.116}$ & $14.5_{-0.493}^{+1.08}$ & $1.14_{-0.00616}^{+0.0255}$ & $4_{-2.54}^{+0.513}$ & $4.33_{-2.21}^{+0.107}$ & $53.7_{-13.3}^{+2.92}$ & $2.18_{-0.0846}^{+0.102}$ & $30.1_{-1.94}^{+11.8}$ & $0.972_{-0.0654}^{+0.00656}$ & $2.38_{-0.536}^{+0.225}$ \\
\hline BP Таu & $-3.97_{-0.155}^{+0.25}$ & $-1.32_{-0.119}^{+0.104}$ & $2.68_{-1.52}^{+0.39}$ & $18.3_{-8.48}^{+3.46}$ & $1.21_{-0.0932}^{+0.0309}$ & $3.33_{-0.236}^{+0.421}$ & $2.56_{-0.716}^{+0.711}$ & $28.5_{-8.78}^{+9.7}$ & $2.79_{-0.733}^{+1.07}$ & $14.2_{-3.71}^{+28}$ & $0.946_{-0.708}^{+0.0252}$ & $1.87_{-0.495}^{+1.46}$ \\
\hline CFHT 4 & $-4.94_{-0.359}^{+0.409}$ & $-0.932_{-0.228}^{+0.089}$ & $1.78_{-0.94}^{+1.14}$ & $8.83_{-3.82}^{+3.56}$ & $1.19_{-0.0747}^{+0.0577}$ & $3.94_{-2.2}^{+0.766}$ & $3.22_{-1.74}^{+1.8}$ & $34.1_{-14.2}^{+13.7}$ & $1.82_{-1.47}^{+0.394}$ & $21.3_{-12.3}^{+20.9}$ & $0.947_{-0.308}^{+0.0444}$ & $1.9_{-1.02}^{+3.41}$ \\
\hline CIDA 1 & $-3.82_{-0.313}^{+0.38}$ & $-0.947_{-0.1}^{+0.0854}$ & $0.801_{-0.185}^{+2.09}$ & $13.9_{-1.11}^{+2.7}$ & $1.07_{-0.0317}^{+0.0202}$ & $4.02_{-1.29}^{+0.985}$ & $4.15_{-2.34}^{+0.978}$ & $36.1_{-11.5}^{+10.4}$ & $2.07_{-0.0107}^{+0.903}$ & $45.3_{-39.1}^{+11.1}$ & $0.0987_{-0.0645}^{+0.831}$ & $4.01_{-2.12}^{+5.03}$ \\
\hline CIDA 7 & $-4.69_{-0.24}^{+0.482}$ & $-1.04_{-0.165}^{+0.096}$ & $2.03_{-0.877}^{+1.23}$ & $11.1_{-1.12}^{+2.19}$ & $1.18_{-0.0245}^{+0.0293}$ & $4.01_{-1.81}^{+1.63}$ & $4.75_{-1.24}^{+0.948}$ & $30.2_{-16.2}^{+21.8}$ & $2.06_{-0.00125}^{+0.297}$ & $17.1_{-8.06}^{+13.1}$ & $0.945_{-0.404}^{+0.0468}$ & $1.13_{-0.391}^{+1.61}$ \\
\hline CIDA 8 & $-4.5_{-0.389}^{+0.421}$ & $-1.24_{-0.189}^{+0.13}$ & $1.93_{-0.642}^{+0.814}$ & $7.86_{-2.65}^{+4.4}$ & $1.21_{-0.0697}^{+0.054}$ & $3.51_{-1.08}^{+0.953}$ & $3.1_{-1.25}^{+0.633}$ & $46.6_{-13.5}^{+13.8}$ & $2.22_{-1.18}^{+2.26}$ & $16.9_{-7.6}^{+12.1}$ & $0.866_{-0.314}^{+0.107}$ & $1.52_{-0.88}^{+2.34}$ \\
\hline CIDA 9 A & $-3.99_{-0.16}^{+0.285}$ & $-1.17_{-0.0844}^{+0.061}$ & $1.77_{-0.448}^{+0.062}$ & $18.1_{-1.65}^{+2.03}$ & $1.1_{-0.0169}^{+0.0162}$ & $3.76_{-0.569}^{+0.791}$ & $3.85_{-0.312}^{+0.44}$ & $38.2_{-5.66}^{+5.52}$ & $2.77_{-0.704}^{+0.832}$ & $17.8_{-7.29}^{+10.5}$ & $0.606_{-0.322}^{+0.256}$ & $1.17_{-0.379}^{+1.1}$ \\
\hline CIDA 12 & $-5.65_{-0.412}^{+0.618}$ & $-1.17_{-0.36}^{+0.137}$ & $1.94_{-1.06}^{+0.999}$ & $10.5_{-4.83}^{+5.82}$ & $1.25_{-0.1}^{+0.0691}$ & $2.89_{-0.747}^{+1.26}$ & $2.97_{-1.36}^{+0.773}$ & $29.7_{-17.8}^{+21.1}$ & $2.71_{-1.83}^{+2.83}$ & $16.3_{-7.42}^{+22.6}$ & $0.974_{-0.205}^{+0.0204}$ & $0.78_{-0.477}^{+1.89}$ \\
\hline CIDA 14 & $-4.76_{-1.38}^{+1.35}$ & $-1.19_{-0.351}^{+0.324}$ & $2.36_{-0.962}^{+0.801}$ & $8.29_{-5.47}^{+6.19}$ & $1.14_{-0.08}^{+0.0713}$ & $4.08_{-1.76}^{+1.7}$ & $2.74_{-0.973}^{+0.748}$ & $50.7_{-21.9}^{+18.2}$ & $1.25_{-1.21}^{+1.84}$ & $13.6_{-6.7}^{+16.2}$ & $0.986_{-0.157}^{+0.0114}$ & $5.21_{-4.05}^{+110}$ \\
\hline
\end{tabular}

Note. Targets marked with an asterisk did not have good fits and were excluded from our demographic analysis.

(This table is available in its entirety in machine-readable form.) 
Table 4

Fit Results (with Size Constraint)

\begin{tabular}{|c|c|c|c|c|c|c|c|c|c|c|c|c|}
\hline Target & $\log \left(M_{\text {dust }} / M_{\odot}\right)$ & $\log \left(r_{\text {in }} / \mathrm{au}\right)$ & $\log \left(r_{c} / \mathrm{au}\right)$ & $H_{100}(\mathrm{au})$ & $\beta$ & $\log \left(a_{\max } / \mu \mathrm{m}\right)$ & $q$ & $i(\mathrm{deg})$ & $\bar{c} \kappa\left(\mathrm{cm}^{2} \mathrm{~g}^{-1}\right)$ & $T_{\text {dust }}(\mathrm{K})$ & $F_{\nu} / F_{\nu, \text { thin }}$ & $M_{\text {dust }} / M_{\text {dust,ana }}$ \\
\hline AA Tau & $-3.95_{-0.12}^{+0.145}$ & $-0.927_{-0.128}^{+0.0776}$ & $1.96_{-0.0258}^{+0.0241}$ & $10.4_{-0.682}^{+0.883}$ & $1.12_{-0.0125}^{+0.0145}$ & $3.46_{-0.367}^{+0.328}$ & $3.29_{-0.188}^{+0.145}$ & $30_{-7.32}^{+7.97}$ & $3.43_{-1.05}^{+1.17}$ & $20.4_{-0.828}^{+0.87}$ & $0.692_{-0.0131}^{+0.0107}$ & $1.18_{-0.279}^{+0.451}$ \\
\hline $\mathrm{AB}$ Aur & $-3.99_{-0.0387}^{+0.0261}$ & $-0.591_{-0.0656}^{+0.0519}$ & $2.29_{-0.0598}^{+0.0407}$ & $13.4_{-0.546}^{+0.578}$ & $1.14_{-0.00909}^{+0.0164}$ & $3.19_{-1.82}^{+1.09}$ & $4.15_{-1.82}^{+0.0995}$ & $44_{-3.91}^{+4.94}$ & $2.3_{-0.218}^{+0.16}$ & $44.2_{-1.4}^{+1.85}$ & $0.888_{-0.013}^{+0.00961}$ & $1.63_{-0.124}^{+0.17}$ \\
\hline ВР Таu & $-3.97_{-0.123}^{+0.174}$ & $-1.27_{-0.101}^{+0.0963}$ & $1.83_{-0.0572}^{+0.0536}$ & $15.5_{-3.24}^{+2.05}$ & $1.18_{-0.0334}^{+0.0206}$ & $3.78_{-0.238}^{+0.27}$ & $2.57_{-0.287}^{+0.351}$ & $35.1_{-11.5}^{+8.72}$ & $1.48_{-0.43}^{+0.578}$ & $27.4_{-2.54}^{+2.13}$ & $0.744_{-0.039}^{+0.0251}$ & $1.99_{-0.474}^{+0.982}$ \\
\hline CFHT 4 & $-5.13_{-0.29}^{+0.385}$ & $-0.927_{-0.108}^{+0.0847}$ & $1.2_{-0.0996}^{+0.103}$ & $12.3_{-4.86}^{+4.76}$ & $1.24_{-0.061}^{+0.0433}$ & $3.94_{-0.866}^{+0.544}$ & $2.57_{-0.902}^{+0.66}$ & $46.1_{-12.3}^{+9.23}$ & $1.05_{-0.643}^{+1.07}$ & $35_{-7.04}^{+5.21}$ & $0.823_{-0.0474}^{+0.0314}$ & $1.17_{-0.541}^{+1.67}$ \\
\hline CIDA 1 & $-4.08_{-0.132}^{+0.115}$ & $-1.01_{-0.105}^{+0.0957}$ & $1.61_{-0.113}^{+0.107}$ & $13_{-0.622}^{+3.20}$ & $1.04_{-0.0161}^{+0.0213}$ & $4.22_{-2.12}^{+1.41}$ & $4.94_{-1.37}^{+0.945}$ & $26.1_{-9.24}^{+9.66}$ & $2.06_{-0.227}^{+0.0126}$ & $17.8_{-2.57}^{+3.15}$ & $0.526_{-0.0944}^{+0.0109}$ & $1.81_{-0.315}^{+0.416}$ \\
\hline CIDA 7 & $-4.84_{-0.13}^{+0.186}$ & $-1.01_{-0.108}^{+0.0706}$ & $1.4_{-0.0986}^{+0.0937}$ & $10.6_{-0.784}^{+2.41}$ & $1.19_{-0.0215}^{+0.0229}$ & $4.51_{-2.52}^{+2.74}$ & $4.8_{-1.04}^{+0.851}$ & $39_{-19.3}^{+16.1}$ & $2.06_{-0.000579}^{+0.108}$ & $25.3_{-2.05}^{+2.68}$ & $0.746_{-0.0714}^{+0.0497}$ & $0.762_{-0.0985}^{+0.117}$ \\
\hline CIDA 8 & $-4.73_{-0.397}^{+0.355}$ & $-1.32_{-0.198}^{+0.131}$ & $1.57_{-0.153}^{+0.12}$ & $8.24_{-2.34}^{+2.98}$ & $1.21_{-0.0473}^{+0.0382}$ & $3.29_{-0.871}^{+0.78}$ & $2.96_{-0.719}^{+0.575}$ & $52_{-13}^{+9.79}$ & $2.85_{-1.4}^{+3.08}$ & $21.8_{-3.13}^{+3.98}$ & $0.713_{-0.0877}^{+0.0569}$ & $0.839_{-0.456}^{+0.883}$ \\
\hline CIDA 9 A & $-4.06_{-0.116}^{+0.158}$ & $-1.18_{-0.0618}^{+0.0998}$ & $1.71_{-0.0542}^{+0.0452}$ & $17.5_{-1.48}^{+1.33}$ & $1.1_{-0.00669}^{+0.007767}$ & $3.89_{-2.39}^{+0.732}$ & $3.85_{-0.683}^{+0.277}$ & $39.9_{-4.1}^{+4.37}$ & $2.52_{-0.446}^{+0.621}$ & $18.3_{-0.768}^{+0.732}$ & $0.58_{-0.0657}^{+0.0358}$ & $1.05_{-0.244}^{+0.462}$ \\
\hline CIDA 12 & $-5.35_{-0.799}^{+1.17}$ & $-1.14_{-0.211}^{+0.133}$ & $1.12_{-0.26}^{+0.239}$ & $11.4_{-5.13}^{+4.74}$ & $1.27_{-0.098}^{+0.0473}$ & $3.73_{-1.37}^{+1.6}$ & $2.18_{-1.03}^{+1.12}$ & $35.9_{-16}^{+15.7}$ & $1.65_{-1.56}^{+2.33}$ & $31.7_{-8.79}^{+8.12}$ & $0.846_{-0.109}^{+0.0697}$ & $0.935_{-0.722}^{+12}$ \\
\hline CIDA 14 & $-4.12_{-1.69}^{+2.37}$ & $-1.19_{-0.397}^{+0.116}$ & $1.19_{-0.521}^{+0.582}$ & $8.91_{-6.67}^{+9.48}$ & $1.18_{-0.112}^{+0.108}$ & $5.7_{-2.56}^{+2.84}$ & $1.97_{-1.16}^{+1.22}$ & $42.4_{-22.4}^{+22.2}$ & $0.0151_{-0.0151}^{+2.05}$ & $47.8_{-32.1}^{+41.1}$ & $0.959_{-0.108}^{+0.0255}$ & $49.7_{-48.4}^{+2.88 e+04}$ \\
\hline
\end{tabular}

Note. Targets marked with an asterisk did not have good fits and were excluded from our demographic analysis.

(This table is available in its entirety in machine-readable form.) 

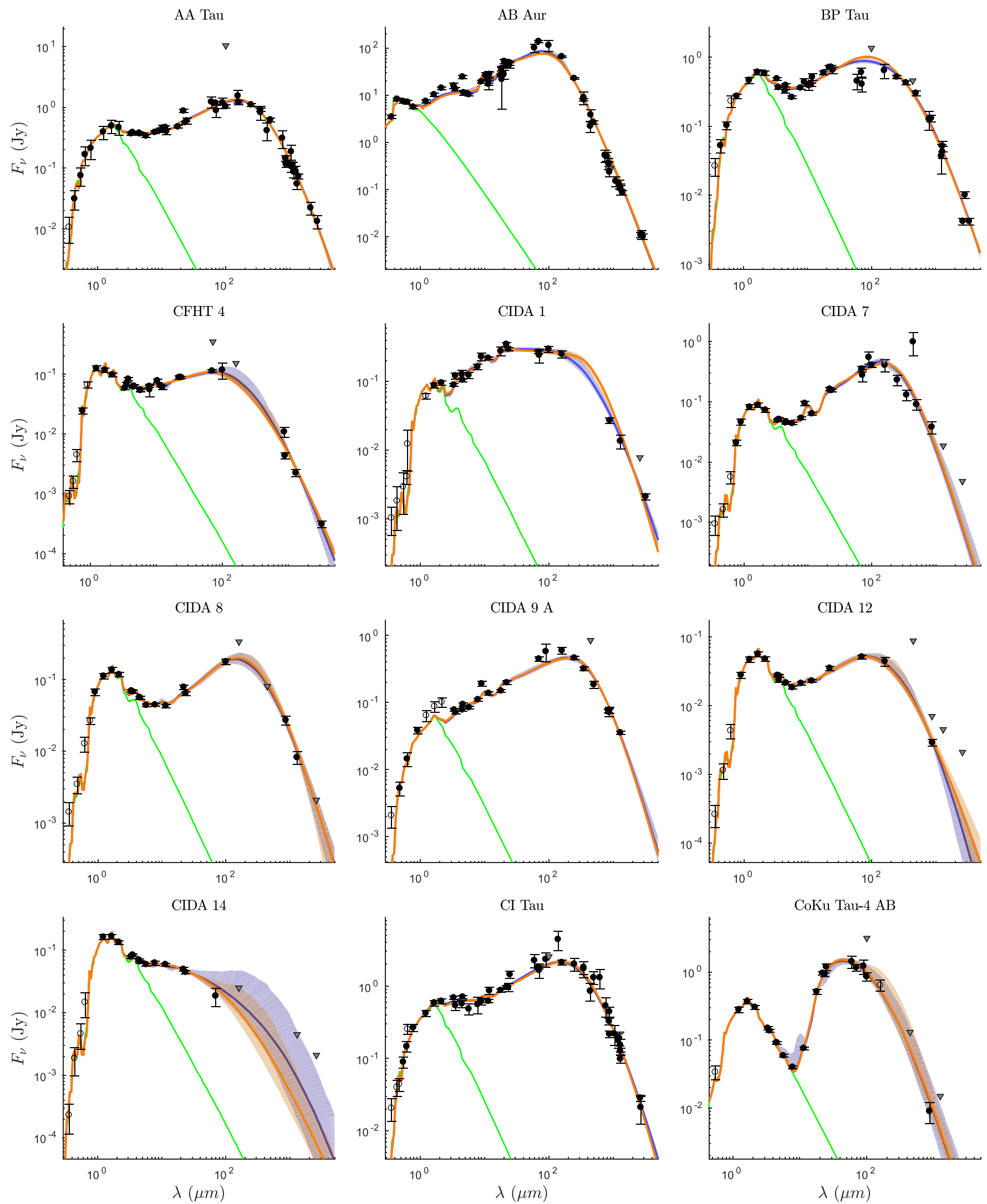

Figure 8. Model fits to the measured SEDs. The data are plotted in black circles (detections) and gray triangles ( $3 \sigma$ upper limits). Open circles are points that were excluded from the fitting. The stellar photosphere is shown in green. The shaded regions show the range (15.9\%-84.1\%) of flux densities from the posterior sample of model fits, with the solid lines showing the median model. Models shown in blue were fit without the size constraint, while models shown in orange were fit with the size constraint. Systems marked with an asterisk were not well fit by our model and were excluded from our subsequent analysis. A subset of our targets is shown here; the complete figure set (11 images) is available in the online journal.

(The complete figure set (11 images) is available.) 

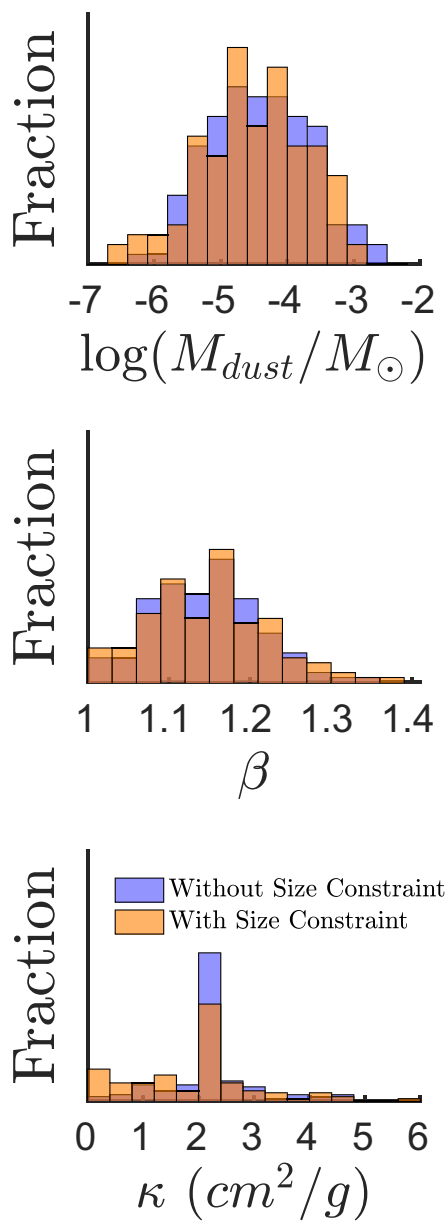
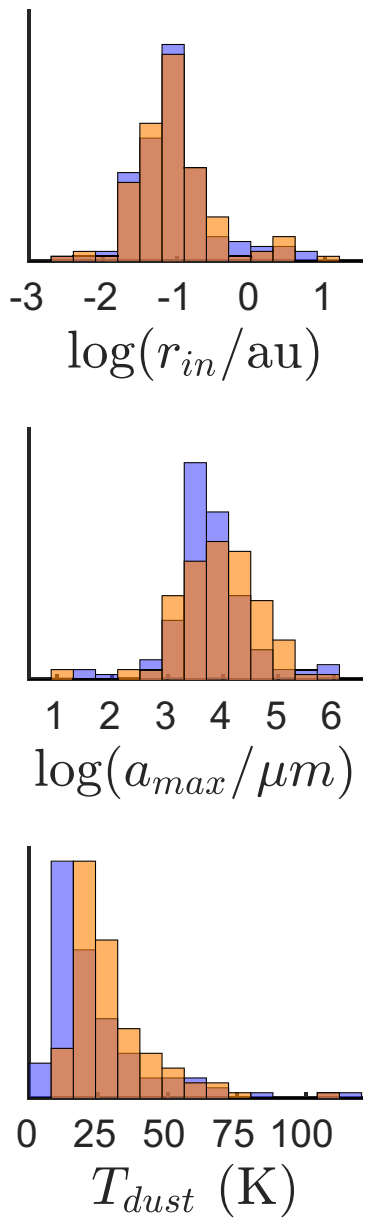
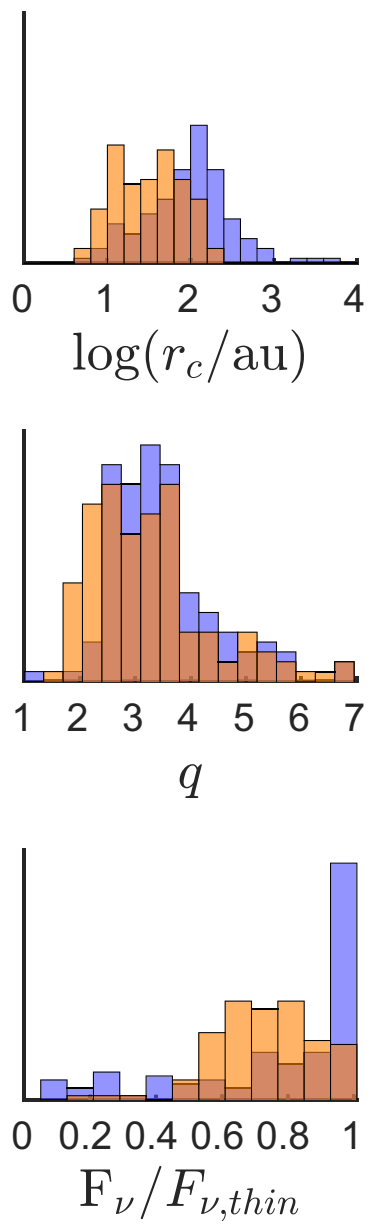
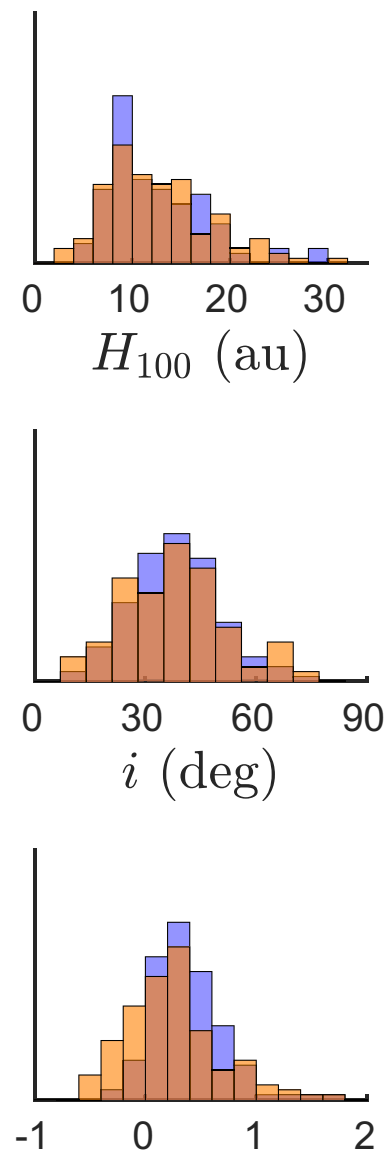

$\log \left(M_{\text {dust }} / M_{\text {dust,ana }}\right)$

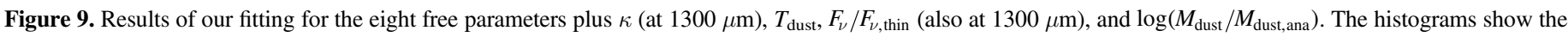

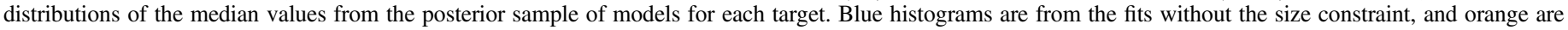
from the fits with the size constraint.

and

$$
A=\overline{\log \left(M_{\text {dust }}\right)}-B \cdot \overline{\log \left(M_{\star}\right)} .
$$

The same formulae were used with $M_{\text {dust }}$ replaced by $F_{\nu}$ to fit for the correlation of flux density with stellar mass. We then examined the distribution of all $10,000 A$ and $B$ values to ascertain the statistical significance of the correlations. The results are listed in Table 5. The samples of fits are shown along with the distributions of eight values in Figures 12 and 13.

We find that the $1300 \mu \mathrm{m}$ flux density does correlate with $M_{\star}$ to a high degree of statistical certainty, and the slope of the relation is roughly linear (or slightly steeper than linear). We are not able to recover a significant correlation between $M_{\text {dust }}$ and $M_{\star}$; a positive correlation is evident only at the $2 \sigma$ level. This is not surprising, considering previous studies mapped disk flux density to dust mass with analytic relations with the error on the flux density measurement as the primary source of uncertainty. Our fitting procedure explored many disk parameters; thus, there were many contributions to our robustly determined dust mass uncertainties.

\section{Summary}

1. We used radiative transfer models to investigate the effect of various parameters on a disk's SED, opacity, temperature, and optical depth in order to better understand the validity of the assumptions commonly used to derive dust masses from (sub-)millimeter flux density measurements.

2. The disk size and dust mass have the largest effects on the temperature and optical depth. Small disks are warmer but more optically thick - competing factors in determining the (sub-)millimeter disk flux density for a given mass.

3. The spectral indices of disks are shallower than would be expected for optically thin emission in the RayleighJeans regime. For small disks, the optical depth effects contribute most to the discrepancy, while for large disks (which are colder), the deviation from the Rayleigh-Jeans approximation contributes most to the discrepancy.

4. We fit radiative transfer models to the SEDs of 132 protoplanetary disks in Taurus. We performed the fits twice, once with unconstrained disk sizes and once with the known disk size-brightness relation imposed. The addition of the size constraint forced the disks to be smaller, warmer, and more optically thick.

5. We found that the dust opacity values are typically peaked around the canonical value of $2.3 \mathrm{~cm}^{2} \mathrm{~g}^{-1}$ at $1300 \mu \mathrm{m}$.

6. The disk temperatures show a trend with $L_{\star}$, as proposed by some previous studies. However, the size-constrained 

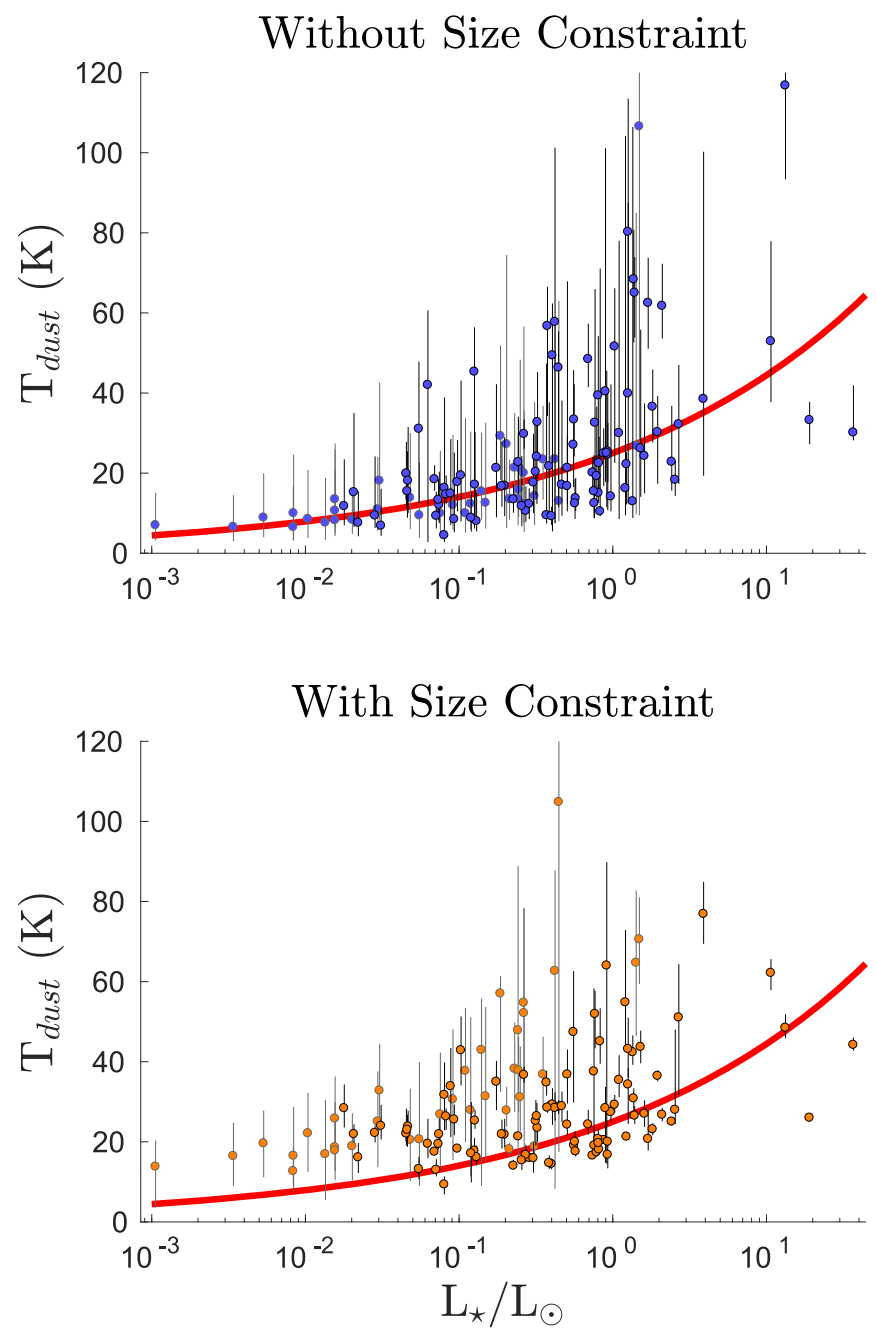

Figure 10. Average dust temperatures of our model disks vs. stellar luminosity. The top panel shows the results with no size constraint, while the bottom panel shows the fits with the size constraint implemented. The points outlined in black and with black error bars are systems with detections at $\lambda>500 \mu \mathrm{m}$, while those in gray have no measurements or only upper limits at $\lambda>500 \mu \mathrm{m}$. The red line is the relation $T_{\text {dust,ana }}=25\left(L_{\star} / L_{\odot}\right)^{1 / 4} \mathrm{~K}$ used by Andrews et al. (2013).

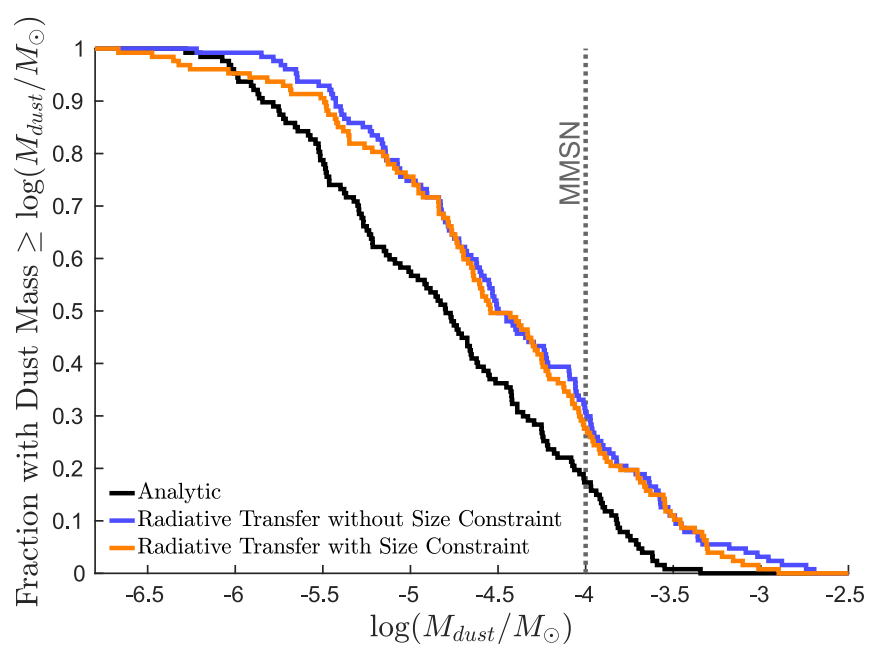

Figure 11. Cumulative distribution of dust masses, i.e., the fraction of disks in our sample with dust masses at least as great as the $x$-axis value. The $M_{\text {dust,ana }}$ values are in black, and our radiative transfer models with and without the imposed size constraint are shown in orange and blue, respectively. The vertical gray dotted line indicates the minimum-mass solar nebula. results have temperatures that are systematically warmer than the previously proposed relations.

7. The radiative transfer model fitting finds the dust masses to be higher than derived using millimeter photometry alone by a factor of $\sim 1-5$. Using the results from radiative transfer models significantly increases the number of disks in the sample with dust masses greater than the minimum-mass solar nebula. This eases the tension between the measured dust masses and the masses of planets in mature systems.

8. We recover the previously found correlation between disk millimeter flux density and stellar mass, but we only measure a positive correlation between dust mass and stellar mass at the $2 \sigma$ confidence level.

We thank Patrick Sheehan for his help and advice on using RADMC-3D and emcee. We also thank the referee for many useful suggestions for improving the manuscript. This material is based upon work supported by the National Aeronautics and Space Administration under agreement No. NNX15AD94G for the program "Earths in Other Solar Systems." The results reported herein benefited from collaborations and/or information exchange within NASA's Nexus for Exoplanet System Science (NExSS) research coordination network sponsored by NASA's Science Mission Directorate. This work was also supported by NSF AAG grant 1311910. An allocation of computer time from the UA Research Computing High Performance Computing (HPC) at the University of Arizona is gratefully acknowledged.

Software: RADMC-3D (Dullemond et al. 2012), emcee (Foreman-Mackey et al. 2013), DIANA Project Opacity Tool (Woitke et al. 2016).

\section{Appendix \\ Notes on Specific Targets}

\section{A.1. AA Tau}

ALMA has revealed multiple radial gaps and rings in this disk (Loomis et al. 2017). Furthermore, the inner part of the disk may be warped (O'Sullivan et al. 2005). Nevertheless, our model (which assumes a smooth radial distribution) fits the SED very well with reasonable parameters, suggesting that these structures do not significantly impact the SED.

\section{A.2. $A B$ Aur}

This disk has an $r<120$ au cavity seen in millimeterwavelength images, and within the cavity, there is an inner dust component and spirals of CO gas (Tang et al. 2012, 2017). Our models cannot account for this complex structure, which may explain the poor fit in the $1-10 \mu \mathrm{m}$ region. We do achieve a good fit at longer wavelengths, suggesting that our model is accurate for the outer disk. There is also a residual envelope of material around this system, although it is unclear if this contributes significantly to the mid-IR flux (Lomax et al. 2016; van der Marel et al. 2016).

\section{A.3. CIDA 1}

Our fits without the size constraint favor a small disk size $\left(r_{c} \sim 6 \mathrm{au}\right)$ in order to match the shape of the SED in the far-IR to submillimeter, although the confidence interval extends to much larger disks. This agrees with the SED fit by 

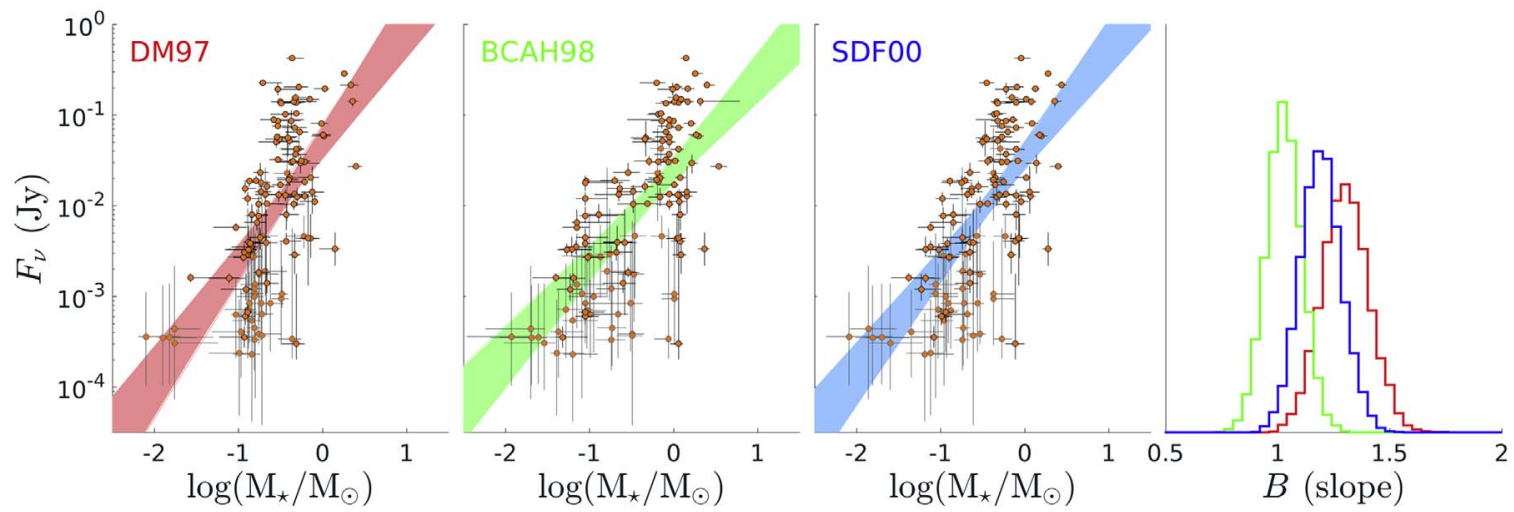

Figure 12. Disk flux density at $1300 \mu \mathrm{m}$ (from our SED fitting with the size constraint implemented) vs. stellar mass. Three different $M_{\star}$ determinations were used, with values taken from Andrews et al. (2013). The points outlined in black and with black error bars are systems with detections at $\lambda>500 \mu \mathrm{m}$, while those in gray have no measurements or only upper limits at $\lambda>500 \mu \mathrm{m}$. The colored regions show the distribution of power-law fits, as described in the main text. The right panel shows the histogram of power-law fit slopes.

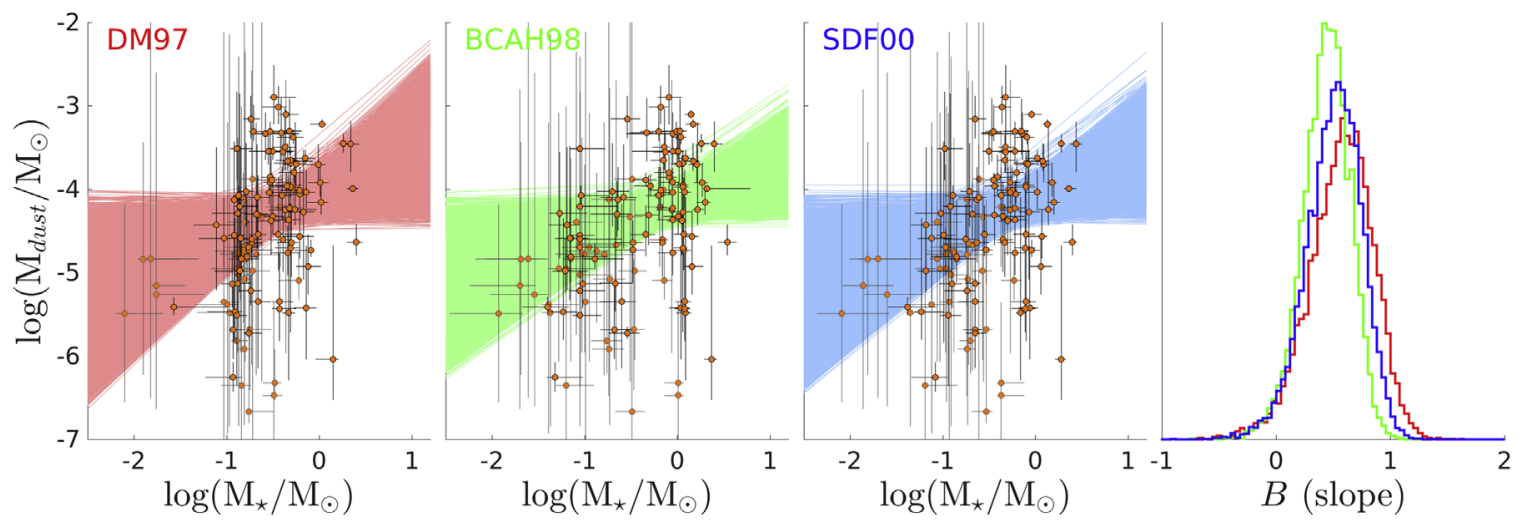

Figure 13. Dust mass (from our SED fitting with the size constraint implemented) vs. stellar mass. Three different $M_{\star}$ determinations were used, with values taken from Andrews et al. (2013). The points outlined in black and with black error bars are systems with detections at $\lambda>500 \mu \mathrm{m}$, while those in gray have no measurements or only upper limits at $\lambda>500 \mu \mathrm{m}$. The colored regions show the distribution of power-law fits, as described in the main text. The right panel shows the histogram of power-law fit slopes.

Table 5

Correlations with Stellar Mass

\begin{tabular}{lcrr}
\hline \hline & DM97 & BCAH98 & SDF00 \\
\hline $\log \left(F_{\nu} /\right.$ Jy $)$ vs. $\log \left(M_{\star} / M_{\odot}\right)$ intercept (A) & $-1.32 \pm 0.0581$ & $-1.61 \pm 0.0381$ & $-1.47 \pm 0.0446$ \\
$\log \left(F_{\nu} /\right.$ Jy) vs. $\log \left(M_{\star} / M_{\odot}\right)$ slope (B) & $1.3 \pm 0.104$ & $1.03 \pm 0.0789$ & $1.19 \pm 0.0905$ \\
$\log \left(M_{\text {dust }} / M_{\odot}\right)$ vs. $\log \left(M_{\star} / M_{\odot}\right)$ intercept (A) & $-4.03 \pm 0.14$ & $-4.16 \pm 0.0909$ & $-4.1 \pm 0.108$ \\
$\log \left(M_{\text {dust }} / M_{\odot}\right)$ vs. $\log \left(M_{\star} / M_{\odot}\right)$ slope (B) & $0.566 \pm 0.284$ & $0.438 \pm 0.21$ & $0.505 \pm 0.245$ \\
\hline
\end{tabular}

Hendler et al. (2017). Including the size constraint forces the disk size to be larger $\left(r_{c} \sim 40 \mathrm{au}\right)$ with a marginally poorer fit to the SED. ALMA observations reveal this source to be a transition disk with a ring of dust peaking at $r \sim 20$ au (Pinilla et al. 2018), although perhaps with a shallow decline in the dust density toward smaller radii, which may explain the observed mid-IR excess. Future work could constrain the disk properties further by fitting the SED and ALMA visibilities simultaneously.

\section{A.4. CIDA 9 A}

CIDA $9 \mathrm{AB}$ is a binary system separated by $2 . ! 3$. (Sub-)millimeter imaging clearly detects emission from $\mathrm{A}$ and places strong upper limits on emission from B (Harris et al. 2012; Akeson \& Jensen 2014); thus, we assume that only A hosts a disk. Although $\mathrm{B}$ is inherently only marginally less luminous than $\mathrm{A}$, it suffers from $\sim 3$ more mag of extinction
(Andrews et al. 2013), so we ignore the contribution from the photosphere of B on the data. We exclude the $J H K$ photometry from our fitting because it appears anomalously bright, perhaps due to the known variability of this object (Furlan et al. 2011).

\section{A.5. CIDA $11 A B$}

This system is a 14.1 au separation binary (Kraus et al. 2012). There is no indication whether the primary or secondary star hosts the disk, or whether both stars do. Thus, we exclude this source from our sample.

\section{A.6. СоКи Тau-3 $A B$}

This system is an $\sim 2^{\prime \prime}$ separation binary system (Kraus et al. 2012) with an ALMA-detected disk around each star (Akeson $\&$ Jensen 2014). The separation is too close for the emission at 
most wavelengths to be resolved and separated into the two disks, so we exclude this source from our sample.

\section{A.7. СоKи Tau-4 AB}

This is an 8 au separation binary system hosting a circumbinary disk (Ireland \& Kraus 2008), so we model the stellar photosphere as a combination of the two stars. The Herschel/PACS $160 \mu \mathrm{m}$ photometry is potentially contaminated by large-scale nebulous emission (Howard et al. 2013), so we exclude it from our fitting.

\section{A.8. CZ Tаи $A B$}

This system is a 0 ". 3 separation binary. There is no indication whether the primary or secondary star hosts the disk, or whether both stars do. Thus, we exclude this source from our sample.

\section{A.9. DD Tau $A B$}

This system is a $0 . ! 3$ separation binary, and it is not clear whether one or both of the stars host a disk (Harris et al. 2012). We exclude this source from our sample.

\section{A.10. DF Tau A}

This system is a 0 "! 1 separation binary system. Following the conclusion by Allen et al. (2017), we assume that only A hosts a disk. We subtracted the stellar flux contribution of B from the data before fitting the SED.

\section{A.11. DG Tau}

This system is very bright in the far-IR, which requires large $H_{100}$ values in our models, although it is also possible that these wavelengths are contaminated by nebulous envelope emission (Nakajima \& Golimowski 1995).

\section{A.12. DH Tau A}

This is a 2". 3 separation binary system, where the secondary is a planetary-mass companion. (Sub-)millimeter emission is detected only from the primary star (Harris et al. 2012; Wolff et al. 2017; Wu et al. 2017), so we model the system with a disk only around A and ignore any contribution to the data from the faint secondary. However, a very compact optically thick disk around the secondary could evade (sub-)millimeter detection (Wu et al. 2017). Our modeling does not fit the data well in the mid-IR, where the SED exhibits a dip that may indicate a pre-transitional disk structure. Thus, we exclude this system from our demographic analysis.

\section{A.13. DK Tau $A B$}

This system is a 3 ". 4 separation binary system with both stars hosting disks (Akeson \& Jensen 2014). The separation is too close for the emission from the two disks to be separated at many wavelengths, so we exclude this source from our sample.

\section{A.14. DP Tau $A B$}

This is a 0" 1 binary system (Kraus et al. 2011). There is no indication whether the primary or secondary star hosts the disk, or whether both stars do. Thus, we exclude this source from our sample.

\section{A.15. DQ Tau $A B$}

This system is a 0.13 au separation spectroscopic binary hosting a circumbinary disk (Czekala et al. 2016). We model the stellar photosphere as a combination of the two stars.

\section{A.16. FM Таu}

Our models struggle somewhat to fit the $1-10 \mu \mathrm{m}$ region of the SED. This suggests that the radial structure in the inner part of the disk may be more complicated than our model's smooth structure.

\section{A.17. FO Tau $A B$}

This is a 22 au separation binary (Kraus et al. 2012). There is no indication whether the primary or secondary star hosts the disk, or whether both stars do. Thus, we exclude this source from our sample.

\section{A.18. FP Tau}

The submillimeter and millimeter photometry detections of this system are clearly discrepant, and our fits effectively split the difference. Additional detections at long wavelengths would yield more decisive results for the grain size parameters.

\section{A.19. FQ Tau $A B$}

This is a $0 . / 75$ separation binary with (sub-)millimeter emission from both stars (Akeson \& Jensen 2014). The contribution to the SED from each disk cannot easily be separated, so we exclude this source from our sample.

\section{A.20. FS Tau $A B$}

This system is a 0 !" 2 separation binary (Harris et al. 2012). We cannot separate contributions to the SED from potential disks around the primary, secondary, or both, so we exclude this source from our sample. For clarity, we note that there is also another star named "FS Tau B" not in our sample and located $\sim 20^{\prime \prime}$ away that hosts an edge-on disk (Kirchschlager et al. 2016).

\section{A.21. FU Tau A and B}

The star FU Tau A is separated from B by 5!"7, which is too close for far-IR observations to isolate the two systems. However, far-IR observations yield only upper limits on flux density, so we apply the same upper-limit constraints to both A and $\mathrm{B}$.

\section{A.22. FV Tau $A B$}

This is a 0 ! 7 separation binary system with both stars likely hosting disks (Harris et al. 2012; Akeson \& Jensen 2014). We cannot isolate the flux from each star at most wavelengths, so we exclude this source from our sample.

\section{A.23. FV Tau-c $A B$}

This is a 0".7 separation binary (Kraus et al. 2012). Both stars appear to host disks, with B being a class I object (McCabe et al. 2006). We cannot isolate the flux from each star at most wavelengths, so we exclude this source from our sample. 


\section{A.24. FX Таи $A B$}

This is a $0 . \prime 9$ separation binary system. Although only A is detected in the (sub-)millimeter (Akeson \& Jensen 2014), midIR observations suggest that B hosts a disk as well (McCabe et al. 2006; Skemer et al. 2011). We cannot isolate the flux from each star at most wavelengths, so we exclude this source from our sample.

\section{A.25. GG Tau A}

This is a triple system where Aa-Ab are separated by 0 !" 24 and $\mathrm{Ab}$ is itself a 4 au binary (Di Folco et al. 2014). The Aa-Ab pair is surrounded by a disk, plus there are smaller circumstellar disks (Dutrey et al. 2016; Yang et al. 2017). Our model is not suitable for this complicated system, so we exclude it from our sample.

\section{A.26. GG Tau B}

This is a binary system with Ba-Bb separated by 1 ". 48 with both stars potentially hosting disks (McCabe et al. 2006). We cannot isolate the flux from each star at most wavelengths, so we exclude this source from our sample.

\section{A.27. GH Tau $A B$}

This is a $0 . ! 3$ binary system with both stars potentially hosting disks (McCabe et al. 2006). We cannot isolate the flux from each star at most wavelengths, so we exclude this source from our sample.

\section{A.28. GM Aur}

The shape of the SED suggests that this system has a pre-transitional disk structure (Hughes et al. 2009; Hornbeck et al. 2016). There are discrepant photometry measurements in the near-to-mid-IR, likely due to variability of the inner disk (Espaillat et al. 2011; Ingleby et al. 2015). Our model is not able to simulate a pre-transitional disk, yet we achieved a good fit to the SED using a combination of low scale height and strong flaring. Thus, the $H_{100}$ and $\beta$ values we derived for this disk should be interpreted with caution.

\section{A.29. GN Tau $A B$}

This is a 0". 4 separation binary with evidence for disks around both stars (Skemer et al. 2011). We cannot isolate the flux from each star at most wavelengths, so we exclude this source from our sample.

\section{A.30. Haro 6-28 $A B$}

This is a 0 !"7 separation binary with both stars possibly hosting disks (McCabe et al. 2006). We cannot isolate the flux from each star at most wavelengths, so we exclude this source from our sample.

\section{A.31. Haro 6-37 $A B$}

This is a 0!' 3 separation binary (Kraus et al. 2012). There is no indication whether the primary or secondary star hosts the disk, or whether both stars do. Thus, we exclude this source from our sample. This system is often referred to as Aab in the literature, with the system listed here as Haro 6-37 C referred to as B.

\section{A.32. Haro 6-37 C}

This star is separated from Haro 6-37 AB by 2" 6. This is too close to isolate the far-IR flux density from these sources, so we simply exclude the far-IR measurements from the fitting. This system is often referred to as B in the literature, with the system listed here as Haro 6-37 AB referred to as Aab.

\section{A.33. HK Tau A}

This star is separated from HK Tau B by 2 !! 3 . This is too close to isolate the far-IR flux density from these sources, so we simply exclude the far-IR measurements from the fitting.

\section{A.34. HK Тau B}

This system hosts an edge-on disk that occults the star and inner disk (Stapelfeldt et al. 1998; McCabe et al. 2011). Thus, we exclude this source from our sample.

\section{A.35. HN Tau A}

This is a binary system with A-B separated by $3^{\prime \prime}$. (Sub-)millimeter images show emission only from A (Harris et al. 2012; Akeson \& Jensen 2014), so we proceed assuming that only the primary star hosts a disk.

\section{A.36. HP Tau}

Some far-IR measurements appear anomalously high, likely due to contamination from nebulous material around this system (Kirk et al. 2013).

\section{A.37. HV Tau C}

This is a well-known edge-on disk (Stapelfeldt et al. 2003; Duchêne et al. 2010), so we exclude it from our sample.

\section{A.38. IRAS $04173+2812$}

This system may be an edge-on disk or a class I source (Luhman et al. 2010; Furlan et al. 2011), so we exclude it from our sample.

\section{A.39. IRAS $04260+2642$}

This source appears to be an edge-on disk (Hartmann et al. 2005; Furlan et al. 2011), so we exclude it from our sample.

\section{A.40. IRAS $04301+2608$}

This system may be an edge-on disk or a class I source (Furlan et al. 2011), so we exclude it from our sample.

\section{A.41. IS Tаи $A B$}

This system is a $0 ! .2$ binary (Schaefer et al. 2014). There is no indication whether the primary or secondary star hosts the disk, or whether both stars do. Thus, we exclude this source from our sample.

\section{A.42. ITG $33 A$}

This is likely an edge-on disk (Andrews et al. 2013), so we exclude it from our sample. 


\section{A.43. IT Tau $A B$}

This is a 2!"4 separation binary system with both stars hosting disks (Harris et al. 2012; Akeson \& Jensen 2014). We cannot isolate the flux from each star at most wavelengths, so we exclude this source from our sample.

\section{A.44. $J 04155799+2746175$}

The single (sub-)millimeter measurement is unusually bright relative to the infrared points, but our models are nevertheless able to fit the SED fairly well. Additional (sub-)millimeter measurements of this source would add clarity.

\section{A.45. $J 04161210+2756385$}

Our models struggle somewhat to fit the near-to-mid-IR region of the SED. This suggests that the radial structure in the inner part of the disk may be more complicated than our model's smooth structure.

\section{A.46. $\mathrm{J} 04202144+2813491$}

This disk is likely edge-on (Furlan et al. 2011; Andrews et al. 2013), so we exclude it from our sample.

\section{A.47. $J 04210795+2702204$}

This source has an anomalously bright IR excess, so we exclude it from our sample.

\section{A.48. $J 04210934+2750368$}

This is a 0".8 separation binary system (Cieza et al. 2012). There is no indication whether the primary or secondary star hosts the disk, or whether both stars do. Thus, we exclude this source from our sample.

\section{A.49. J04213459+2701388}

We are not able to achieve a satisfactory fit to this source, so we exclude it from our demographic analysis. The Herschel/ PACS $160 \mu \mathrm{m}$ photometry may be contaminated by nebulous emission (Bulger et al. 2014), so we exclude this point from our fitting. Our model does fit the $70 \mu \mathrm{m}$ point but does not fit the mid-IR data well, nor does it agree with the strong (sub-)millimeter upper limit. This may indicate that the $70 \mu \mathrm{m}$ photometry is contaminated as well.

\section{A.50. $J 04284263+2714039 A B$}

This is a 0 ". 6 separation binary system with both stars perhaps hosting disks (Kraus \& Hillenbrand 2009). We cannot isolate the flux from each star at most wavelengths, so we exclude this source from our sample.

\section{A.51. J04290068+2755033}

The stellar temperature, luminosity, and extinction for this source are not given by Andrews et al. (2013). We use values of $T_{*}=2700 \mathrm{~K}, L_{*}=0.01043 L_{\odot}$, and $A_{v}=1.71$ from Liu et al. (2015).

\section{A.52. $J 04324938+2253082$}

This source, also known as JH $112 \mathrm{~B}$, is a $0 . " 5$ binary with evidence for disks around both stars (Akeson \& Jensen 2014).
We cannot isolate the flux from each star at most wavelengths, so we exclude this source from our sample.

\section{A.53. $J 04330945+2246487$}

The (sub-)millimeter detection of this disk appears anomalously bright, so we exclude it from our fit. Even so, we are not able to fit the SED satisfactorily with our models, so we exclude this target from our demographic analysis.

\section{A.54. $J 04381486+2611399$}

This source hosts an edge-on disk (Luhman et al. 2007), so we exclude it from our sample.

\section{A.55. $J 04390396+2544264$}

The submillimeter and millimeter photometry detections appear somewhat discrepant. Additional detections at long wavelengths would yield more decisive results for the grain size parameters.

\section{A.56. $J 04403979+2519061 A B$}

This system is an $\sim 7$ au binary (Kraus et al. 2012), so we model the system as a circumbinary disk.

\section{A.57. $J 04414489+2301513$}

This source includes the Bab components of a larger quadruple system (Bowler \& Hillenbrand 2015). The Ba-Bb separation is 0 " 1 . There is no indication whether the primary or secondary star hosts the disk, or whether both stars do. Thus, we exclude this source from our sample.

\section{A.58. $J 04414825+2534304$}

We note that the SED data file provided by Andrews et al. (2013) is erroneously labeled as "J04414825+2523118."

\section{A.59. JH 112 A}

This is a binary system with Aa-Ab separated by 1 !" 5 and both stars hosting disks (Harris et al. 2012; Akeson \& Jensen 2014). We cannot isolate the flux from each star at many wavelengths, so we exclude this source from our sample.

\section{A.60. JH $223 \mathrm{AB}$}

This is a $2^{\prime \prime}$ separation binary system with evidence that both stars host disks (Itoh et al. 2015). We cannot isolate the flux from each star at many wavelengths, so we exclude this source from our sample.

\section{A.61. JH 56}

The SED of this source resembles that of a debris disk instead of a class II protoplanetary disk.

\section{A.62. KPNO 3}

The submillimeter and millimeter photometry detections appear somewhat discrepant. Additional detections at long wavelengths would yield more decisive results for the grain size parameters. 


\section{A.63. KPNO 10}

Several SED data points around $\sim 4 \mu \mathrm{m}$ are anomalously low, and we exclude them from the fitting.

\section{A.64. LkHa 267}

This system either is class I or hosts an edge-on disk (Andrews et al. 2013), so we exclude it from our sample.

\section{A.65. $M H O 2 A B$}

This system is a 7.3 au binary (Kraus et al. 2011), so we model the system as a circumbinary disk.

\section{A.66. $\mathrm{MHO} 3 \mathrm{AB}$}

This system is a 4.5 au binary (Kraus et al. 2011), so we model the system as a circumbinary disk.

\section{A.67. $R W$ Aur $A B$}

This is a 1."4 binary system where both stars host disks (Harris et al. 2012). We cannot isolate the flux from each star at many wavelengths, so we exclude this source from our sample.

\section{A.68. St 34 ABC}

In this system, $\mathrm{AB}$ forms a tight binary that is separated from C by 1."2 (Kraus et al. 2011). We do not know which of the stars host disks, so we exclude this source from our sample.

\section{A.69. T Таи N}

This system is separated from T Tau Sab by 0 ."7, and all three stars host disks (Ratzka et al. 2009). We cannot isolate the flux of $\mathrm{T}$ Tau $\mathrm{N}$ at many wavelengths, so we exclude it from our sample.

\section{A.70. UX Tau A}

Though part of a triple system, UX Tau A is the only one of the three stars with a disk (McCabe et al. 2006). The shape of the SED in the mid-IR suggests that this may be a pre-transitional disk (Espaillat et al. 2010). Our model is not suitable for such a radial structure, and our fit is very poor. Thus, we exclude this source from our demographic analysis.

\section{A.71. $U Y$ Aur $A B$}

This is a $0 . / 9$ separation binary with evidence for circumstellar disks around each star (Akeson \& Jensen 2014), as well as a circumbinary disk (Close et al. 1998). We cannot separate the flux from each disk at most wavelengths, so we exclude this source from our sample.

\section{A.72. UZ Tаи E}

This is a spectroscopic binary, which we model as a circumbinary disk around $\mathrm{Ea}+\mathrm{Eb}$. It is separated from UZ Tau W by 3". 6 , which is sufficient to separate the emission from the disks at most wavelengths, except in the far-IR (Howard et al. 2013).

\section{A.73. UZ Tau W}

This is a 0 ". 4 separation binary with both stars likely hosting disks (Harris et al. 2012). We cannot isolate the flux from each star at most wavelengths, so we exclude this source from our sample.

\section{A.74. V410 X-ray $7 \mathrm{AB}$}

This is a 4.6 au binary (Kraus et al. 2011), so we model the system as a circumbinary disk.

\section{A.75. V807 Tau A}

This system is separated from V807 Tau Bab by 0." 3, but the Bab components do not host disks (Schaefer et al. 2012). The contribution to SED data from the Bab photosphere emission has been subtracted from the measurements.

\section{A.76. V892 Tаи АB}

This is a 7 au binary system (Smith et al. 2005), so we model it as a circumbinary disk. We found a bimodal population of model fits; one population could fit the near-to-mid-IR SED, while the other could fit the far-IR-to-millimeter SED. No models could fit the whole SED well, so we exclude this source from our demographic analysis.

\section{A.77. V955 Tau AB}

This is a 0." 3 separation binary (Kraus et al. 2011) with evidence that both stars host disks (McCabe et al. 2006). We cannot isolate the flux from each star at most wavelengths, so we exclude this source from our sample.

\section{A.78. VY Tau $A B$}

This is a 0"7 separation binary (Kraus et al. 2011). There is no indication whether the primary or secondary star hosts the disk, or whether both stars do. Thus, we exclude this source from our sample.

\section{A.79. XEST 26-062}

The submillimeter and millimeter photometry detections of this system are clearly discrepant, and our fits effectively split the difference. Additional detections at long wavelengths would yield more decisive results for the grain size parameters.

\section{A.80. XZ Tau $A B$}

This is a 0!" 3 separation binary (Kraus et al. 2011). We do not know which of the stars host disks, so we exclude this source from our sample.

\section{A.81. ZZ Tau $A B$}

This is a 6 au binary system (Kraus et al. 2011), but it does not host a circumbinary disk (Espaillat et al. 2012). We do not know which of the stars host disks, so we exclude this source from our sample.

\section{A.82. ZZ Tau IRS}

This system likely hosts an edge-on disk (Furlan et al. 2011; Bulger et al. 2014), so we exclude it from our sample.

\section{ORCID iDs}

Nicholas P. Ballering (1D https://orcid.org/0000-00024276-3730

Josh A. Eisner (10 https://orcid.org/0000-0002-1031-4199 


\section{References}

Akeson, R. L., Ciardi, D. R., van Belle, G. T., \& Creech-Eakman, M. J. 2002, ApJ, 566, 1124

Akeson, R. L., \& Jensen, E. L. N. 2014, ApJ, 784, 62

Allen, T. S., Prato, L., Wright-Garba, N., et al. 2017, ApJ, 845, 161

ALMA Partnership, Brogan, C. L., Pérez, L. M., et al. 2015, ApJL, 808, L3

Andrews, S. M., Rosenfeld, K. A., Kraus, A. L., \& Wilner, D. J. 2013, ApJ, 771,129

Andrews, S. M., Terrell, M., Tripathi, A., et al. 2018, ApJ, 865, 157

Andrews, S. M., \& Williams, J. P. 2005, ApJ, 631, 1134

Andrews, S. M., Wilner, D. J., Hughes, A. M., Qi, C., \& Dullemond, C. P. 2010, ApJ, 723, 1241

Ansdell, M., Williams, J. P., Manara, C. F., et al. 2017, AJ, 153, 240

Baraffe, I., Homeier, D., Allard, F., \& Chabrier, G. 2015, A\&A, 577, A42

Beckwith, S. V. W., \& Sargent, A. I. 1991, ApJ, 381, 250

Beckwith, S. V. W., Sargent, A. I., Chini, R. S., \& Guesten, R. 1990, AJ, 99, 924

Bergin, E. A., \& Williams, J. P. 2018, arXiv:1807.09631

Bowler, B. P., \& Hillenbrand, L. A. 2015, ApJL, 811, L30

Broekhoven-Fiene, H., Matthews, B., Duchêne, G., et al. 2014, ApJ, 789, 155

Brunngräber, R., Wolf, S., Ratzka, T., \& Ober, F. 2016, A\&A, 585, A100

Buckle, J. V., Drabek-Maunder, E., Greaves, J., et al. 2015, MNRAS, 449, 2472

Bulger, J., Patience, J., Ward-Duong, K., et al. 2014, A\&A, 570, A29

Carpenter, J. M., Ricci, L., \& Isella, A. 2014, ApJ, 787, 42

Castelli, F., \& Kurucz, R. L. 2004, arXiv:astro-ph/0405087

Chiang, E. I., \& Goldreich, P. 1997, ApJ, 490, 368

Cieza, L. A., Olofsson, J., Harvey, P. M., et al. 2013, ApJ, 762, 100

Cieza, L. A., Schreiber, M. R., Romero, G. A., et al. 2012, ApJ, 750, 157

Clanton, C., \& Gaudi, B. S. 2014, ApJ, 791, 91

Close, L. M., Dutrey, A., Roddier, F., et al. 1998, ApJ, 499, 883

Czekala, I., Andrews, S. M., Torres, G., et al. 2016, ApJ, 818, 156

Daemgen, S., Natta, A., Scholz, A., et al. 2016, A\&A, 594, A83

D’Alessio, P., Calvet, N., \& Hartmann, L. 2001, ApJ, 553, 321

D’Alessio, P., Calvet, N., Hartmann, L., Franco-Hernández, R., \& Servín, H. 2006, ApJ, 638, 314

D’Alessio, P., Calvet, N., Hartmann, L., Lizano, S., \& Cantó, J. 1999, ApJ, 527,893

Desch, S. J. 2007, ApJ, 671, 878

Di Folco, E., Dutrey, A., Le Bouquin, J.-B., et al. 2014, A\&A, 565, L2

Dorschner, J., Begemann, B., Henning, T., Jaeger, C., \& Mutschke, H. 1995, A\&A, 300, 503

Duchêne, G., McCabe, C., Pinte, C., et al. 2010, ApJ, 712, 112

Dullemond, C. P., \& Dominik, C. 2004, A\&A, 417, 159

Dullemond, C. P., Juhasz, A., Pohl, A., et al. 2012, RADMC-3D: A Multipurpose Radiative Transfer Tool Astrophysics Source Code Library, ascl:1202.015

Dutrey, A., Di Folco, E., Beck, T., \& Guilloteau, S. 2016, A\&ARv, 24, 5

Dutrey, A., Guilloteau, S., Duvert, G., et al. 1996, A\&A, 309, 493

Duvert, G., Guilloteau, S., Ménard, F., Simon, M., \& Dutrey, A. 2000, A\&A, 355,165

Eisner, J. A., Arce, H. G., Ballering, N. P., et al. 2018, ApJ, 860, 77

Eisner, J. A., Bally, J. M., Ginsburg, A., \& Sheehan, P. D. 2016, ApJ, 826, 16

Espaillat, C., D’Alessio, P., Hernández, J., et al. 2010, ApJ, 717, 441

Espaillat, C., Furlan, E., D’Alessio, P., et al. 2011, ApJ, 728, 49

Espaillat, C., Ingleby, L., Hernández, J., et al. 2012, ApJ, 747, 103

Foreman-Mackey, D., Hogg, D. W., Lang, D., \& Goodman, J. 2013, PASP, 125,306

Furlan, E., Luhman, K. L., Espaillat, C., et al. 2011, ApJS, 195, 3

Gräfe, C., Wolf, S., Roccatagliata, V., Sauter, J., \& Ertel, S. 2011, A\&A, 533, A89

Haisch, K. E., Jr., Barsony, M., Ressler, M. E., \& Greene, T. P. 2006, AJ, 132,2675

Hardy, A., Caceres, C., Schreiber, M. R., et al. 2015, A\&A, 583, A66

Harris, R. J., Andrews, S. M., Wilner, D. J., \& Kraus, A. L. 2012, ApJ, 751,115

Hartmann, L., Calvet, N., Gullbring, E., \& D’Alessio, P. 1998, ApJ, 495, 385

Hartmann, L., Megeath, S. T., Allen, L., et al. 2005, ApJ, 629, 881

Hayashi, C. 1981, PThPS, 70, 35

Hendler, N. P., Mulders, G. D., Pascucci, I., et al. 2017, ApJ, 841, 116

Hildebrand, R. H. 1983, QJRAS, 24, 267

Hornbeck, J. B., Swearingen, J. R., Grady, C. A., et al. 2016, ApJ, 829, 65

Howard, C. D., Sandell, G., Vacca, W. D., et al. 2013, ApJ, 776, 21

Huang, J., Öberg, K. I., Qi, C., et al. 2017, ApJ, 835, 231
Hughes, A. M., Andrews, S. M., Espaillat, C., et al. 2009, ApJ, 698, 131 Ingleby, L., Espaillat, C., Calvet, N., et al. 2015, ApJ, 805, 149

Ireland, M. J., \& Kraus, A. L. 2008, ApJL, 678, L59

Itoh, Y., Fukagawa, M., Shibai, H., Sumi, T., \& Yamamoto, K. 2015, PASJ, 67,88

Jensen, E. L. N., Koerner, D. W., \& Mathieu, R. D. 1996, AJ, 111, 2431

Jensen, E. L. N., Mathieu, R. D., \& Fuller, G. A. 1994, ApJL, 429, L29

Kirchschlager, F., Wolf, S., \& Madlener, D. 2016, MNRAS, 462, 858

Kirk, J. M., Ward-Thompson, D., Palmeirim, P., et al. 2013, MNRAS, 432, 1424

Kraus, A. L., \& Hillenbrand, L. A. 2009, ApJ, 704, 531

Kraus, A. L., Ireland, M. J., Hillenbrand, L. A., \& Martinache, F. 2012, ApJ, 745,19

Kraus, A. L., Ireland, M. J., Martinache, F., \& Hillenbrand, L. A. 2011, ApJ, 731,8

Kwon, W., Looney, L. W., Mundy, L. G., \& Welch, W. J. 2015, ApJ, 808, 102

Lada, C. J. 1987, in IAU Symp. 115, Star-forming Regions, ed. M. Peimbert \& J. Jugaku (Dordrecht: Reidel), 1

Liu, Y., Joergens, V., Bayo, A., Nielbock, M., \& Wang, H. 2015, A\&A, 582, A22

Lomax, J. R., Wisniewski, J. P., Grady, C. A., et al. 2016, ApJ, 828, 2

Loomis, R. A., Öberg, K. I., Andrews, S. M., \& MacGregor, M. A. 2017, ApJ, 840,23

Luhman, K. L., Adame, L., D’Alessio, P., et al. 2007, ApJ, 666, 1219

Luhman, K. L., Allen, P. R., Espaillat, C., Hartmann, L., \& Calvet, N. 2010, ApJS, 186, 111

Lynden-Bell, D., \& Pringle, J. E. 1974, MNRAS, 168, 603

Marton, G., Calzoletti, L., Perez Garcia, A. M., et al. 2017, arXiv:1705.05693

Mathieu, R. D., Stassun, K., Basri, G., et al. 1997, AJ, 113, 1841

Maucó, K., Briceño, C., Calvet, N., et al. 2018, ApJ, 859, 1

McCabe, C., Duchêne, G., Pinte, C., et al. 2011, ApJ, 727, 90

McCabe, C., Ghez, A. M., Prato, L., et al. 2006, ApJ, 636, 932

McClure, M. 2009, ApJL, 693, L81

Min, M., Dullemond, C. P., Kama, M., \& Dominik, C. 2011, Icar, 212, 416

Min, M., Hovenier, J. W., \& de Koter, A. 2005, A\&A, 432, 909

Min, M., Rab, C., Woitke, P., Dominik, C., \& Ménard, F. 2016, A\&A, 585, A13

Miotello, A., van Dishoeck, E. F., Kama, M., \& Bruderer, S. 2016, A\&A, 594, A85

Miyake, K., \& Nakagawa, Y. 1993, Icar, 106, 20

Miyake, K., \& Nakagawa, Y. 1995, ApJ, 441, 361

Mohanty, S., Greaves, J., Mortlock, D., et al. 2013, ApJ, 773, 168

Mulders, G. D., \& Dominik, C. 2012, A\&A, 539, A9

Najita, J. R., \& Kenyon, S. J. 2014, MNRAS, 445, 3315

Nakajima, T., \& Golimowski, D. A. 1995, AJ, 109, 1181

O’Sullivan, M., Truss, M., Walker, C., et al. 2005, MNRAS, 358, 632

Osterloh, M., \& Beckwith, S. V. W. 1995, ApJ, 439, 288

Pascual, N., Montesinos, B., Meeus, G., et al. 2016, A\&A, 586, A6

Pascucci, I., Testi, L., Herczeg, G. J., et al. 2016, ApJ, 831, 125

Pérez, L. M., Chandler, C. J., Isella, A., et al. 2015, ApJ, 813, 41

Phan-Bao, N., Lee, C.-F., Ho, P. T. P., Dang-Duc, C., \& Li, D. 2014, ApJ, 795, 70

Phan-Bao, N., Lee, C.-F., Ho, P. T. P., \& Tang, Y.-W. 2011, ApJ, 735, 14

Piétu, V., Guilloteau, S., Di Folco, E., Dutrey, A., \& Boehler, Y. 2014, A\&A, 564, A95

Pinilla, P., Benisty, M., Birnstiel, T., et al. 2014, A\&A, 564, A51

Pinilla, P., Natta, A., Manara, C. F., et al. 2018, A\&A, 615, A95

Pinilla, P., Quiroga-Nuñez, L. H., Benisty, M., et al. 2017, ApJ, 846, 70

Pollack, J. B., Hollenbach, D., Beckwith, S., et al. 1994, ApJ, 421, 615

Ratzka, T., Schegerer, A. A., Leinert, C., et al. 2009, A\&A, 502, 623

Rebull, L. M., Padgett, D. L., McCabe, C.-E., et al. 2010, ApJS, 186, 259

Ribas, Á, Espaillat, C. C., Macías, E., et al. 2017, ApJ, 849, 63

Ricci, L., Testi, L., Natta, A., et al. 2014, ApJ, 791, 20

Ricci, L., Trotta, F., Testi, L., et al. 2012, A\&A, 540, A6

Rodriguez, J. E., Pepper, J., Stassun, K. G., et al. 2015, AJ, 150, 32

Salter, D. M., Kóspál, Á, Getman, K. V., et al. 2010, A\&A, 521, A32

Sawicki, M. 2012, PASP, 124, 1208

Schaefer, G. H., Prato, L., Simon, M., \& Patience, J. 2014, AJ, 147, 157

Schaefer, G. H., Prato, L., Simon, M., \& Zavala, R. T. 2012, ApJ, 756, 120

Schulz, B., Marton, G., Valtchanov, I., et al. 2017, arXiv:1706.00448

Sheehan, P. D., \& Eisner, J. A. 2018, ApJ, 857, 18

Skemer, A. J., Close, L. M., Greene, T. P., et al. 2011, ApJ, 740, 43

Skinner, S. L., Brown, A., \& Walter, F. M. 1991, AJ, 102, 1742

Smith, K. W., Balega, Y. Y., Duschl, W. J., et al. 2005, A\&A, 431, 307

Stapelfeldt, K. R., Krist, J. E., Ménard, F., et al. 1998, ApJL, 502, L65 
Stapelfeldt, K. R., Ménard, F., Watson, A. M., et al. 2003, ApJ, 589, 410

Tang, Y.-W., Guilloteau, S., Dutrey, A., et al. 2017, ApJ, 840, 32

Tang, Y.-W., Guilloteau, S., Piétu, V., et al. 2012, A\&A, 547, A84

Tazzari, M., Testi, L., Ercolano, B., et al. 2016, A\&A, 588, A53

Tazzari, M., Testi, L., Natta, A., et al. 2017, A\&A, 606, A88

Testi, L., Birnstiel, T., Ricci, L., et al. 2014, in Protostars and Planets VI, ed. H. Beuther et al. (Tucson, AZ: Univ. Arizona Press), 339

Testi, L., Natta, A., Shepherd, D. S., \& Wilner, D. J. 2001, ApJ, 554, 1087

Tripathi, A., Andrews, S. M., Birnstiel, T., et al. 2018, ApJ, 861, 64

Tripathi, A., Andrews, S. M., Birnstiel, T., \& Wilner, D. J. 2017, ApJ, 845, 44

van der Marel, N., Verhaar, B. W., van Terwisga, S., et al. 2016, A\&A, 592, A126 van der Plas, G., Ménard, F., Ward-Duong, K., et al. 2016, ApJ, 819, 102

Ward-Duong, K., Patience, J., Bulger, J., et al. 2018, AJ, 155, 54

Weidenschilling, S. J. 1977, Ap\&SS, 51, 153

Williams, J. P., \& Best, W. M. J. 2014, ApJ, 788, 59

Woitke, P., Min, M., Pinte, C., et al. 2016, A\&A, 586, A103

Wolff, S. G., Ménard, F., Caceres, C., et al. 2017, AJ, 154, 26

Wu, Y.-L., Close, L. M., Eisner, J. A., \& Sheehan, P. D. 2017, AJ, 154,234

Yang, Y., Hashimoto, J., Hayashi, S. S., et al. 2017, AJ, 153, 7

Zubko, V. G., Mennella, V., Colangeli, L., \& Bussoletti, E. 1996, MNRAS, 282, 1321 


\title{
Erratum: "Protoplanetary Disk Masses from Radiative Transfer Modeling: A Case Study in Taurus" (2019, AJ, 157, 144)
}

\author{
Nicholas P. Ballering (iD) and Josh A. Eisner (D) \\ Steward Observatory, University of Arizona, 933 North Cherry Avenue, Tucson, AZ 85721, USA; ballerin@email.arizona.edu \\ Received 2019 April 22; published 2019 May 6
}

There was a plotting error in Figure 5 of the original paper. The blue curves in the third column were plotted using too few points so the shapes of the curves were not clear. A corrected version of the figure is shown here.
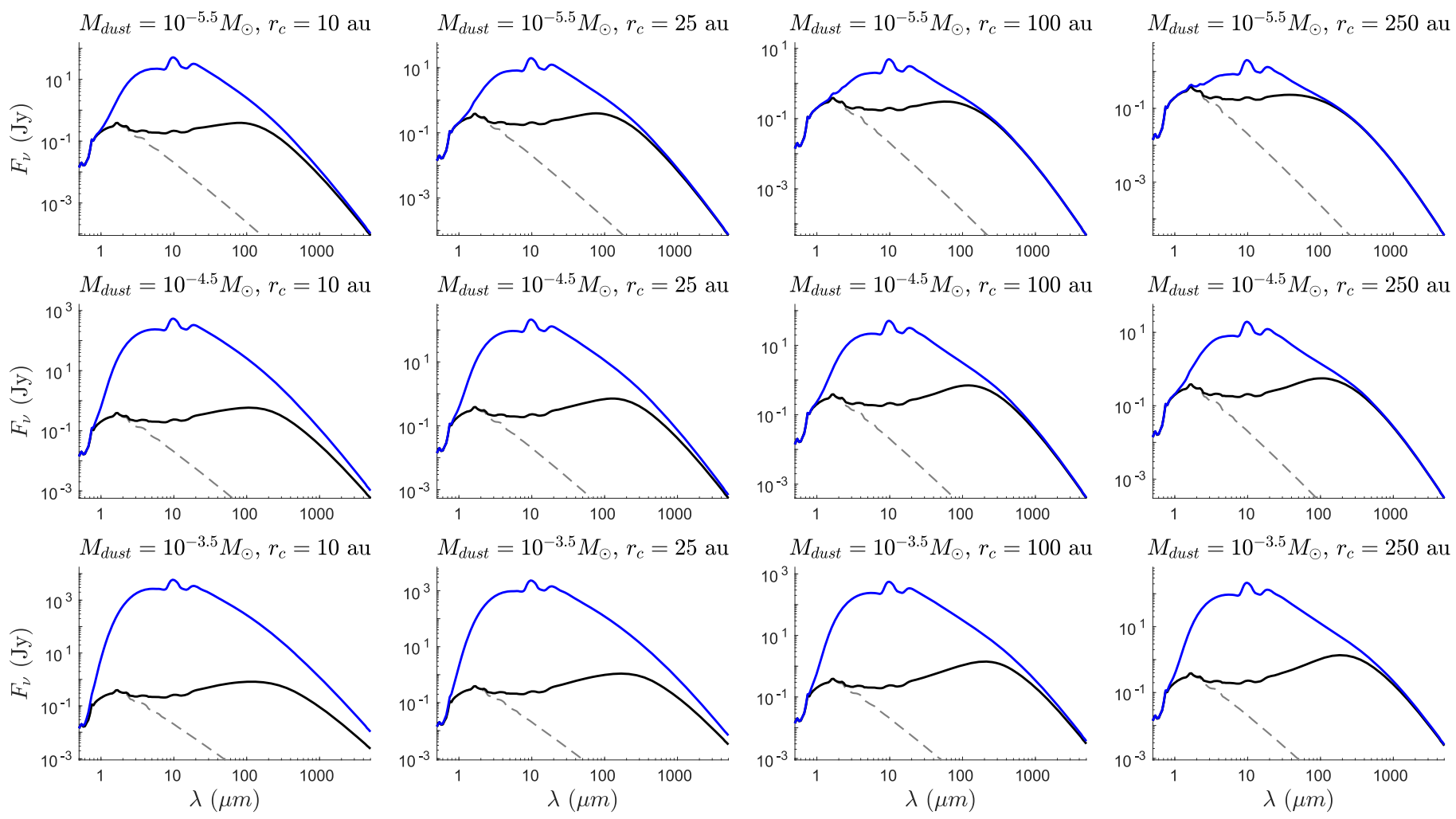

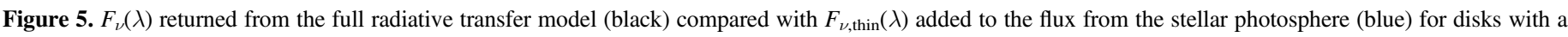
variety of dust masses (rows) and disk sizes (columns). The gray dashed line in each plot is the stellar photosphere.

\section{ORCID iDs}

Nicholas P. Ballering (iD https://orcid.org/0000-0002-4276-3730

Josh A. Eisner (D) https://orcid.org/0000-0002-1031-4199 